\title{
Combined zero degree structure beam dynamics and applications
}

\author{
Ulrich Ratzinger, Hendrik Hähnel ${ }^{*}$, Rudolf Tiede, and Jan Kaiser \\ Institute for Applied Physics, Goethe University, Max-von-Laue-Strasse 1, \\ 60438 Frankfurt am Main, Germany \\ Ali Almomani \\ Physics Department, Faculty of Science, Yarmouk University, 211-63 Irbid, Jordan
}

(Received 29 May 2019; published 12 November 2019)

\begin{abstract}
The combined zero degree structure (KONUS) is a quasiperiodic structure. It was developed for the low-energy part of multigap drift tube linacs with H-type cavities. Their rf efficiency depends very much on a low electrical capacity of the drift tube structure, while in E-type structures like the Alvarez-DTL this is a minor effect. Therefore, instead of having quadrupole singlets integrated in voluminous drift tubes, KONUS allows one to develop a separated function drift tube linac (DTL) with a large voltage gain between two lenses. Very low beam injection energies can be realized, as the drift tube lengths can range down to around $10 \mathrm{~mm}$. One KONUS period consists of a triplet lens, a rebuncher with a few gaps at a synchronous phase around $-35^{\circ}$, and the main multigap acceleration designed for a hypothetical zero degree synchronous particle. The longitudinal beam dynamics along this main acceleration section and the layout of the quadrupole triplet channel are explained in detail. Two examples for pulsed high current proton and heavy ion acceleration are included.
\end{abstract}

DOI: 10.1103/PhysRevAccelBeams.22.114801

\section{INTRODUCTION}

The Kombinierte Null Grad Struktur (KONUS) beam dynamics [1-3] has been developed since 1980. A first step was the distinction of a bunch center particle and of the synchronous particle for zero degree structures with a defined number of gaps [4]. In a next step, a combination of two zero degree sections was realized successfully at the $\mathrm{cw}$ operated $10 \mathrm{MV}$ postaccelerator behind the $13 \mathrm{MV}$ HV Tandem of the Munich accelerator laboratory [5]. In 1991, three KONUS periods with two internal quadrupole triplets [6] were realized at the High Charge State Injector HLI at GSI Darmstadt [7]. The longitudinal acceptance was increased by rebunching sections behind each triplet lens in order to accept an radio frequency quadrupole (RFQ) beam. Figure 1 shows a view into that cavity, while Fig. 2 explains the periodicity of the KONUS dynamics. This was the first RFQ-IH-DTL combination with an energy range of the IH cavity from $300 \mathrm{~A} \mathrm{keV} \mathrm{up}$ to $1.4 \mathrm{~A} \mathrm{MeV}$ and at $\mathrm{A} / \mathrm{q}$ values up to 8.5 , corresponding to $\mathrm{U}^{28+}$ from the Caprice ECR source [8]. The duty factor

\footnotetext{
"haehnel@iap.uni-frankfurt.de
}

Published by the American Physical Society under the terms of the Creative Commons Attribution 4.0 International license. Further distribution of this work must maintain attribution to the author(s) and the published article's title, journal citation, and DOI. is up to $50 \%$. The GSI-HLI has been in routine operation since 1991. The standard operation mode is beam injection into the Unilac Alvarez section at 25\% duty factor.

Similar RFQ-IH-DTL linacs are in operation now at many places: (i) CERN, Geneva (linac3, 4.2 A MeV lead

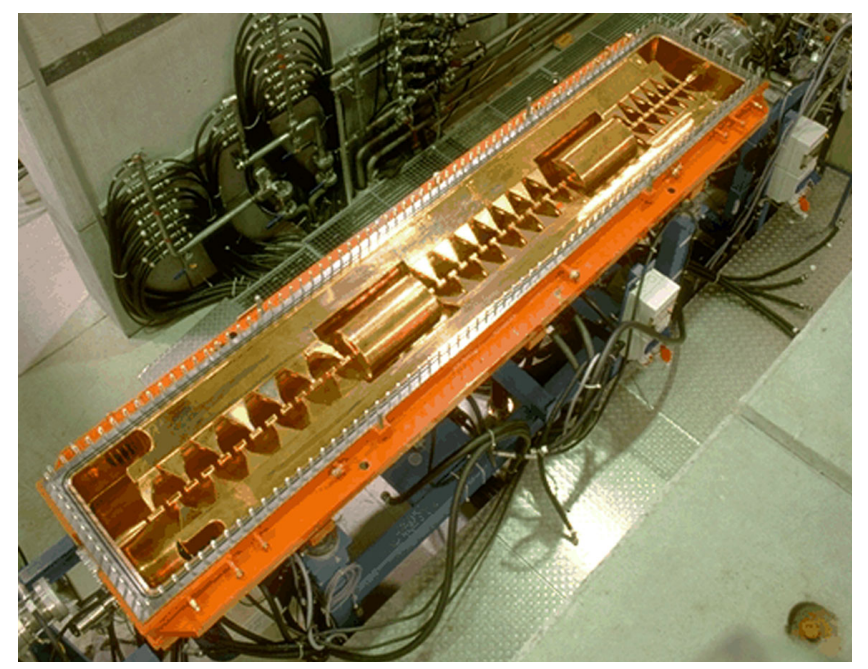

FIG. 1. View on the $108 \mathrm{MHz}, 9.4 \mathrm{MV}$ GSI-HLI cavity after taking away the upper half shell. The IH-type drift tube structure shows three KONUS sections with two internal quadrupole triplet lenses. The water cooling distribution needed for $50 \%$ duty factor operation is visible in the background. 


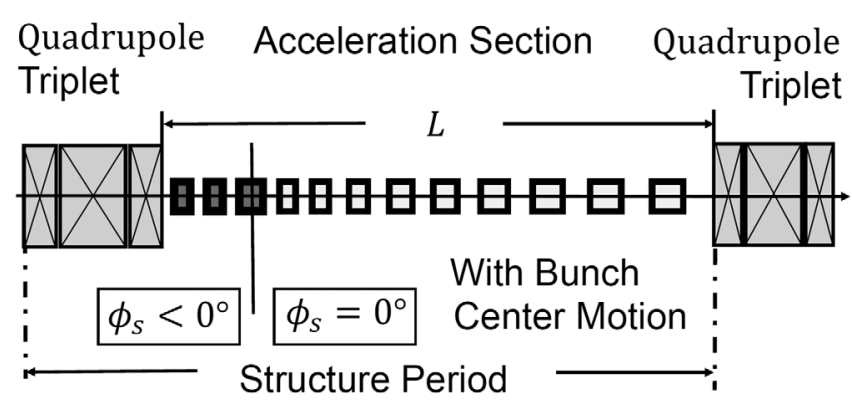

FIG. 2. Scheme of a KONUS lattice period: Transverse focusing is obtained by quadrupole lenses (here FDF-O-FDF-O lattice); longitudinal focusing and acceleration are achieved by a rebunching section at constant negative synchronous phase, followed by a zero degree section with the bunch injected at a slightly higher energy.

ions [9,10], Rex-Isolde [11]), (ii) BNL, Brookhaven (electron beam ion source linac, 3.2 A MeV gold ions [12]), (iii) medical synchrotron injectors for $\mathrm{C}^{4+}$ (HIT Heidelberg [13], CNAO Pavia, MIT Marburg, SPHIC Shanghai, and Medaustron Wiener Neustadt), (iv) JINR, Dubna (Hilac operable and Lilac under construction for the future Nuclotron-based Ion Collider Facility [14]), (v) GSI, Darmstadt (HITRAP decelerator linac [15]), (vi) TRIUMF, Vancouver (ISAC linac1 [16]), and (vii) GSI, Darmstadt (high current injector HSI for $\mathrm{U}^{4+}$ beams $\left.[17,18]\right)$.

A next step is the application of KONUS for high-current proton acceleration [19]. Detailed design studies, cavity development, and ordering as well as production of components for the 3-68 MeV, $70 \mathrm{~mA}$ facility for antiproton and ion research (FAIR) proton CH-DTL were done at IAP Frankfurt and at GSI Darmstadt during the past $20 \mathrm{yr}$.

In the case of CERN linac3, an Alvarez design as an alternative to the IH-DTL was completed and is documented by two CERN "yellow reports" $[20,21]$. They show very clearly the pros and cons of each layout.

Figure 3 underlines the argument for the use of H-type structures with KONUS beam dynamics: It is the high shunt impedance. At the same time, experiments confirmed that H-type cavities with slim drift tubes can reach an unprecedented voltage gain per meter of $10 \mathrm{MV} / \mathrm{m}$ at $\beta$ values as low as 0.07 [22].

These projects demonstrate the wide range of useful applications for KONUS. In the following, descriptions and results from qualitative and quantitative investigations on that beam dynamics are given, with a main focus on the KONUS zero degree section and its integration into the periodic structure.

In the case of a high current, high duty factor proton, and deuteron acceleration (like needed for intense neutron sources, for example), beam halo formation plays an important role. In such cases, well-aligned DTLs of the Alvarez type based on a negative synchronous phase
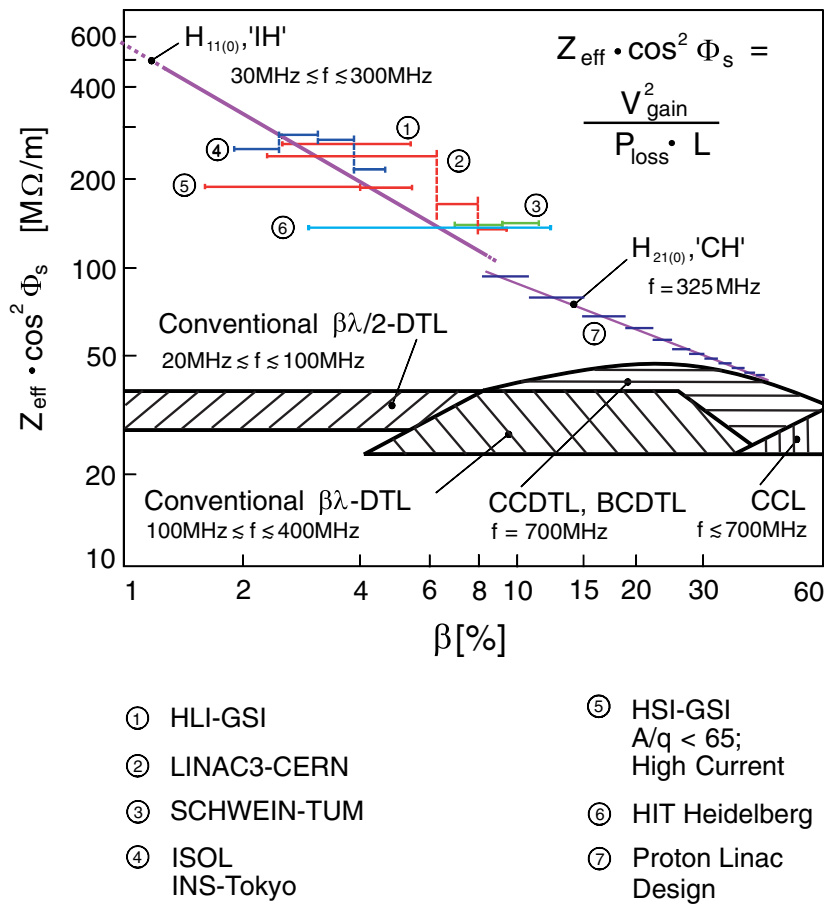

(5) $\mathrm{HSI}-\mathrm{GSI}$ $\mathrm{A} / \mathrm{q}<65$ High Current

(6) HIT Heidelberg

(7) Proton Linac Design

FIG. 3. Shunt impedance plot of DTL structures for low $\beta$ and up to around $100 \mathrm{~A} \mathrm{MeV}$. The H-type structures ( $\mathrm{IH}$ and $\mathrm{CH}$ cavities) are very efficient up to $100 \mathrm{~A} \mathrm{MeV}$ beam energy. $L$ denotes the cavity length. $\phi_{c, i}$ denotes the synchronous phase of the bunch in gap no. $i$ (compare Secs. II and III), while $\left\langle\phi_{c}\right\rangle$ is the effective value-averaged over all gaps of a cavity.

structure (NSPS) and with FODO or FOFODODO ${ }^{1}$ quadrupole focusing show advantages in beam quality. KONUS structures will need further improvement and an advanced collimator concept to be ready for applications of that kind. One KONUS layout study for $150 \mathrm{~mA}$ deuteron beams and with superconducting magnetic solenoid focusing and superconducting $\mathrm{CH}$ cavities was already performed successfully and seems promising [23].

Section II explains the motion of the bunch center particle around the zero degree synchronous particle. The latter one defines the drift tube array. The gap number dependence of each zero degree section from the entrance energy, acceleration rate, and excess energy of the bunch center, as well as longitudinal phase advance and bunch half axis ratios, are discussed in Sec. III. Section IV describes the transverse beam dynamics in quadrupole triplet channels, while, finally, Sec. V shows two KONUS high current design cases including error simulations by applying the simulation codes LORASR (see the Appendix) and TRACEWIN.

\footnotetext{
${ }^{1}$ Convenient way of expressing the polarity along a quadrupole channel [3]. Where F: Focusing Element; D: Defocusing Element; O: Drift Element.
} 


\section{LONGITUDINAL PARTICLE DYNAMICS ALONG THE ZERO DEGREE SECTION}

\section{A. Overview}

One KONUS structure period is shown in Fig. 2. The bunch preparation in transverse phase space by a quadrupole triplet and in longitudinal phase space by a few-gap rebuncher section is followed by the main acceleration section-a zero degree multigap structure.

The longitudinal acceptance of a zero degree synchronous phase structure of infinite length is vanishing. At a given cell number, however, such structures show a welldefined phase-space acceptance. Figures 4(a) and 4(b) illustrate the single-particle motion around the zero degree synchronous particle (SP).

Along the second quadrant, the single-particle motion around the SP is quite smooth. Particles with a surplus in

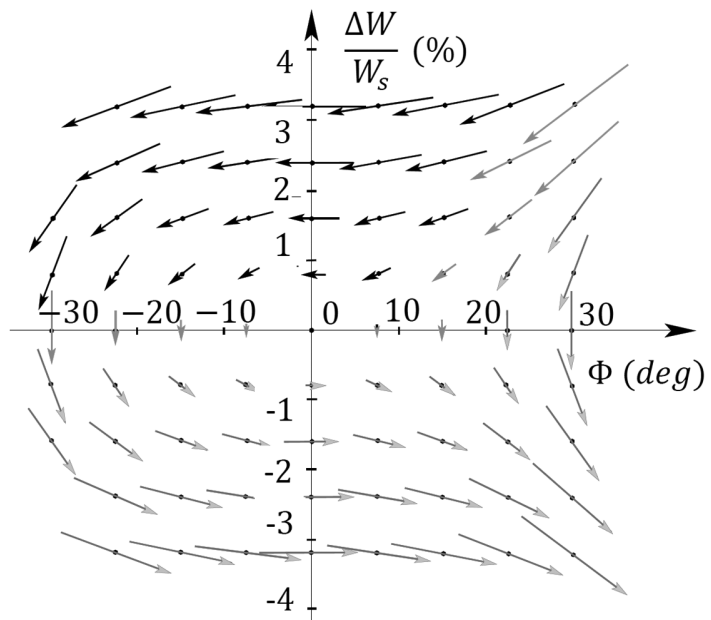

(a)

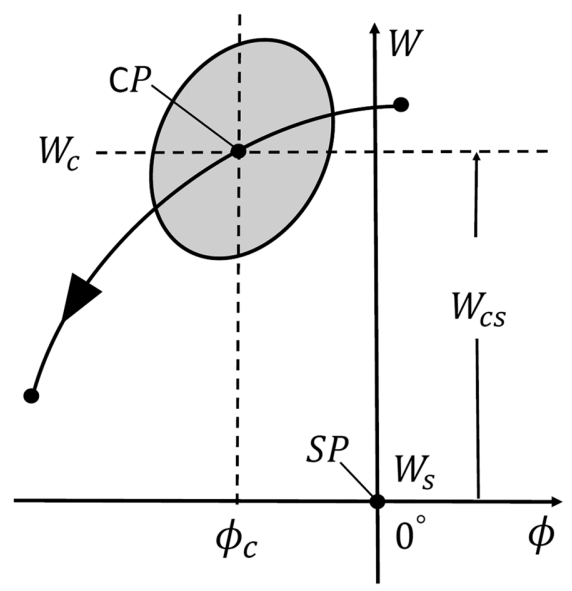

(b)

FIG. 4. (a) Single-particle motion around the zero degree synchronous particle. (b) Typical trajectory of the bunch center along a zero degree KONUS drift tube section with a limited number of cells. injection energy relative to the zero degree synchronous particle are moving towards negative rf phases. Before the particle's extra energy is exhausted, the zero degree section is ended. The following KONUS section will start again with a triplet lens followed by a rebuncher. The starting SP energy of the next zero degree structure is defined in a way that the bunch center delivered by the rebuncher will again have a surplus in energy to move along the second quadrant in energy phase space.

This explains the KONUS beam dynamics' similarity to most linac structures in the sense that lenses (quadrupole doublets, triplets, or solenoids in the case of superconducting linacs) are combined with lens-free drift tube sections, operated at a negative synchronous particle phase. The zero degree section is helpful at lower beam energies with a high relative energy gain per KONUS period, where traditionally the FODO focusing concept [3] is applied along multicell cavities of the Wideroe or of the Alvarez type with quadrupole singlets installed in every (or in a few cases every second) drift tube.

In KONUS, the ions pass the gaps in the central region between two lenses at relatively small negative phase angles. This reduces the growth in beam diameter, caused by the defocusing gap field action which is proportional to $\sin \Phi$ [3], where $\Phi$ denotes the phase angle of the ion when passing the gap midplane. If, in contrast, one tries to apply a constant but small negative synchronous phase along the same drift tube section, the longitudinal focusing will become increasingly nonlinear, as this force is proportional to $\cos \Phi-\cos \Phi_{s}$. In KONUS, the phase angles immediately in front of and behind each lens are as negative as needed for given beam parameters, such as the emittance and beam current, while only in the central part are phases close to zero degree.

There is a general rule applicable for the length of focusing lenses: The shorter these elements are, the larger is the provided longitudinal beam acceptance of the KONUS structure - as no longitudinal focusing is provided along the lenses.

Finally, just the zero degree section has to be described in detail, as everything else is quite conventional in KONUS.

\section{B. Equations of motion in the $W \phi$ plane}

The longitudinal dynamics is defined by the entrance beam energy, by the synchronous particle energy for each cell, and by the linac frequency. A Wideroe-type drift tube lattice is assumed with $L_{i}=\beta_{i} \cdot \lambda / 2$. During one-half $\mathrm{rf}$ period $T / 2$, one cell is passed, with an energy gain $G$ for the zero degree synchronous particle at each gap center (Fig. 5). The time is given in units of $T / 2$ :

$$
t_{i}^{\prime}=t_{0}^{\prime}+i
$$

with 


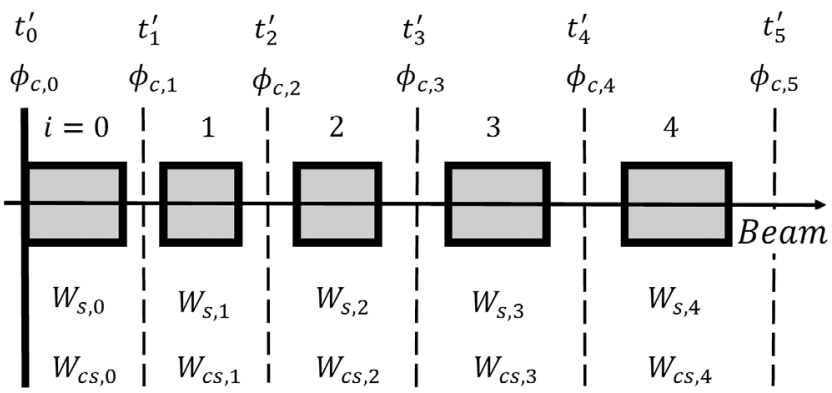

FIG. 5. Scheme of a drift tube structure with terms and positions.

$$
t_{0}^{\prime}=\frac{W_{s}\left(t_{0}^{\prime}\right)}{G}=\frac{W_{s, 0}}{G}
$$

and

$$
\Delta t^{\prime}=1
$$

corresponding to a time step $\frac{T}{2}=\frac{\pi}{\omega}$.

The integer $i$ corresponds to the time $t_{i}^{\prime}$, when the synchronous particle is passing the midplane of gap number $i$. At the starting point of the calculation, which is just defined at a distance $\beta_{0} \cdot \lambda / 2$ in front of the first gap, $i=0$ is valid (see Fig. 5). $W_{s, i}$ denotes the zero degree synchronous particle energy in cell number $i$. Along the acceleration section, the following equations apply for the synchronous particle:

$$
\begin{gathered}
W_{s, i}=G \cdot t_{i}^{\prime}, \\
\gamma_{s, i}=1+\frac{W_{s, i}}{m_{0} c^{2}}, \\
\phi_{s}=0^{0}=\text { const. }
\end{gathered}
$$

Now the motion of the bunch center particle relative to the zero degree synchronous structure particle will be described. The energy is assumed to be changed in one step in the gap midplane. Between two midplanes $i$ and $i+1$, the phase $\phi_{c}$ of the center particle is shifted:

$$
\Delta \phi_{c}=-\pi \cdot \frac{\beta_{c}-\beta_{s}}{\beta_{s}} .
$$

With the relation

$$
\frac{d \gamma}{d \beta}=\gamma^{3} \cdot \beta
$$

one gets for small $\delta \beta$

$$
\Delta \phi_{c}=-\pi \cdot \frac{\delta \beta}{\beta_{s}} \cong-\pi \cdot \frac{\delta \gamma}{\gamma_{s}^{3} \cdot \beta_{s}^{2}} .
$$

$\delta \gamma$ is related to $W_{c s}$ by

$$
W_{c s}=\delta \gamma \cdot m_{0} c^{2}
$$

Inserting $\delta \gamma$ from Eq. (10) into Eq. (9) results in a phase shift

$$
\phi_{c, i+1}-\phi_{c, i}=-\pi \cdot \frac{W_{c s, i}}{\gamma_{s, i} \cdot\left(\gamma_{s, i}+1\right) \cdot G \cdot t_{i}^{\prime}}
$$

between gap centers $i$ and $i+1$ and in a change of the energy difference $W_{c s, i}$ across gap $i$ of

$$
W_{c s, i+1}-W_{c s, i}=G \cdot\left[\cos \left(\phi_{c, i+1}\right)-1\right] .
$$

Figure 6 shows as an example nine paths of optional center particles (CPs) around the zero degree synchronous $\mathrm{SP}$ in the case of ten accelerating gaps. In this example, the SP energy for protons was increased along the acceleration section by a factor of 2.1-from 2.7 to $5.7 \mathrm{MeV}$. When a particle is crossing the trajectory of a neighbored particle, it is always time (and energy) shifted at the crossing point (this also applies to Fig. 8).

For the same case, Fig. 7 shows a contour plot of particle exit energies against their starting conditions in phase and energy. Neighbored contour lines differ by $1 \%$ of the SP exit energy. There exists a longitudinal injection phasespace area within the first quadrant, where particles will show a surplus in energy against the zero degree SP down to the exit. This plot gives an idea of the longitudinal acceptance of such a ten-gap section, depending in which energy-phase area the bunch is injected and which exit energy spread will be tolerated. Moreover, for a given value of the injected emittance, the ellipse orientation has to be

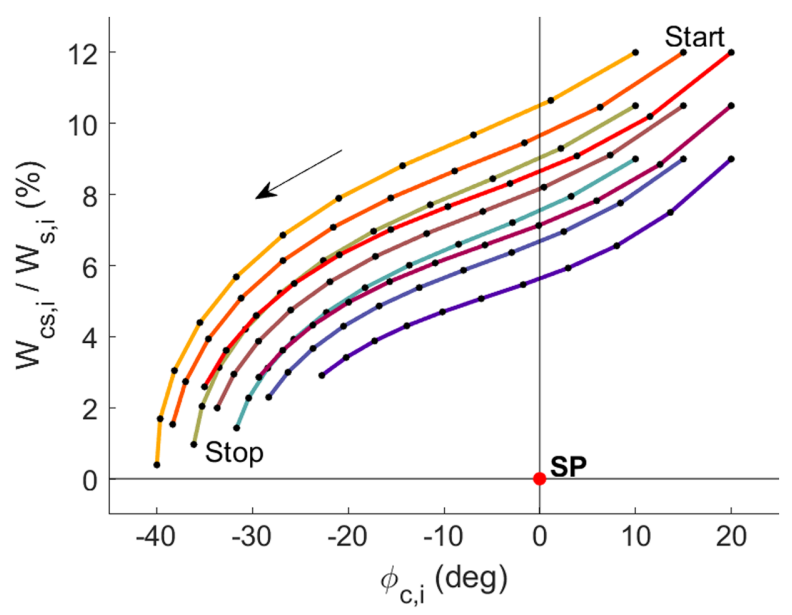

FIG. 6. Optional center particles' paths around the synchronous particle for a ten-gap KONUS section. The ordinate shows the relative energy deviation against the SP with its energy increasing in each gap. Dots mark gap centers. 


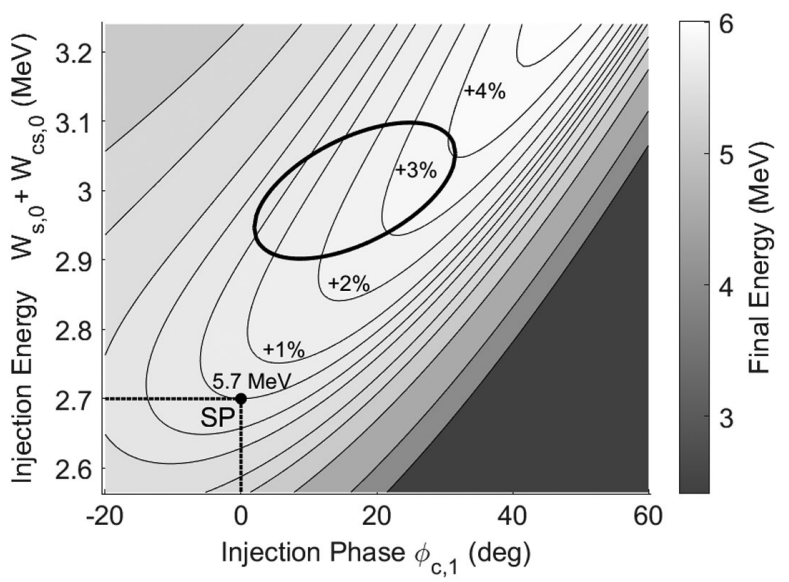

FIG. 7. Exit energy contour plot on the input energy-phase plane. The ellipse marks a preferable injection phase-space area, which results in small emittance growth, small transverse defocusing, and acceptable final energy spread (see Fig. 9).

matched for minimum emittance growth along the zero degree section.

This is demonstrated by Fig. 8 for the example of particles placed on a parallelogram around the central particle. In the plotted case, the transformed phase space shows a compact form and only low geometric distortions. The phase advance (rotation of the parallelogram in phase space) is well below $90^{\circ}$. Figure 9 shows the longitudinal rms emittance growth for the same case when injecting a particle array within an ellipse. These results were obtained by using a custom-written MATLAB transport code employing the above formulated transport equations. One can clearly see that the optimum is reached for negative values of the Twiss parameter $\alpha$, in agreement with the orientation of the parallelogram $a b c d$ in Fig. 8.

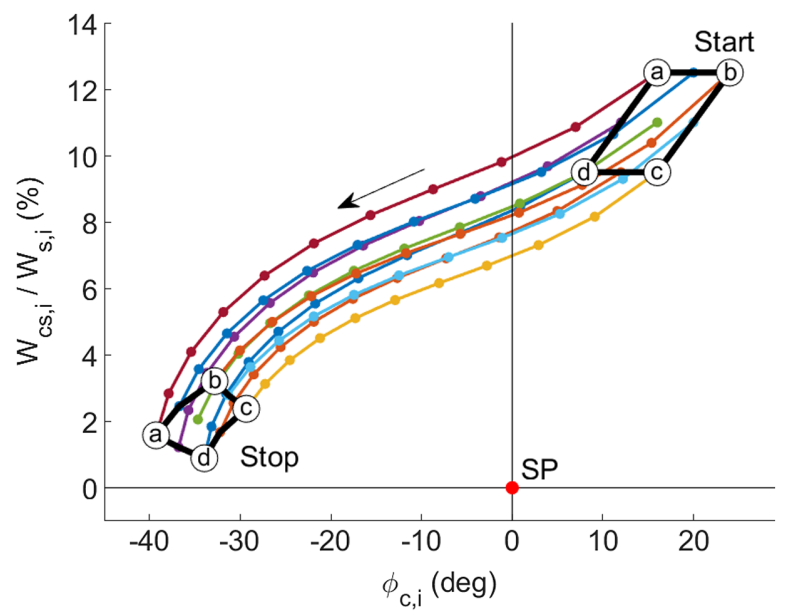

FIG. 8. Transformation of a well-oriented area abcd along the ten-gap acceleration section. Note that the energy deviation of the bunch centers is shown in percent of the steadily increasing SP energy along the section.

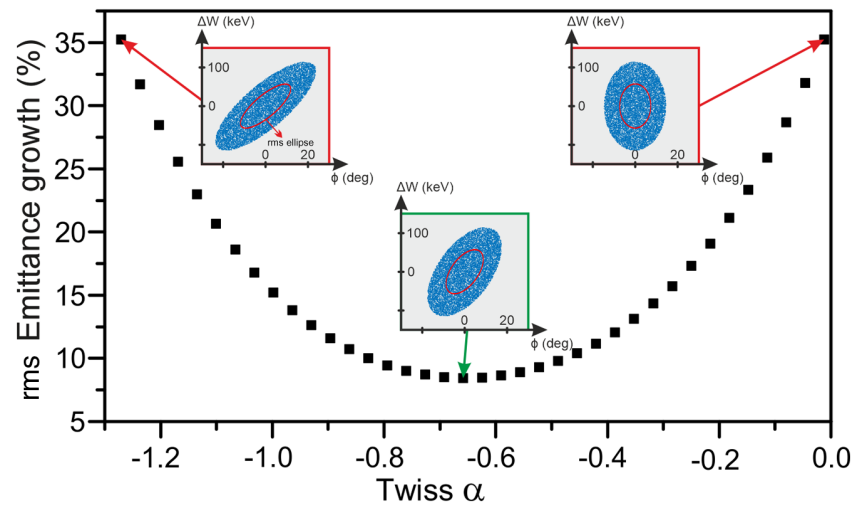

FIG. 9. Simulated emittance growth rate for emittance areas injected at different Twiss parameters $\alpha$.

\section{THE ZERO DEGREE SECTION IN NONRELATIVISTIC APPROXIMATION}

\section{A. The bunch center motion}

The main advantage of KONUS lies at the low-energy end of a DTL, where the absolute transverse beam emittances restrict the cell number of conventional lensfree cavities to low values like 2-4 in the case of coaxial quarter and half wave resonators. KONUS allows gap numbers from 8 and up to above 20 between focusing lenses, as described below.

When compared to FODO accelerator structures with internal quadrupole lenses, lower injection energies at a given operation frequency become accessible with KONUS. With modern production technologies, this advantage might even be further exploited in the future for low beam current applications. In this case, the RFQ in front of the DTL can become shorter, which will save rf power and acceleration length.

In the following, the zero degree section is investigated analytically in more detail by starting from Eqs. (11) and (12) and by applying several approximations.

In the nonrelativistic limit and employing a Taylor expansion to the cosine function at reasonably small angles below $25^{\circ}$, the particle motion is described by

$$
\begin{aligned}
& \dot{\phi}_{c}=-\frac{\pi}{2} \cdot \frac{W_{c s}}{G \cdot t^{\prime}}, \\
& \dot{W}_{c s}=-\frac{G}{2} \cdot \phi_{c}^{2} .
\end{aligned}
$$

As explained earlier, the phase space of interest in the $W \phi$ plane is the second quadrant mainly, with positive $W_{c s}$ and negative (up to slightly positive) $\phi_{c}$. As no general solutions for this set of differential equations were found, a Taylor expansion for $W_{c s}$ around $W_{c s, 0} \geq 0, \phi_{c, 0}=0$ was performed: 


$$
\begin{aligned}
W_{c s}\left(t^{\prime}\right)= & W_{c s, 0}\left[1-\frac{\pi^{2} \cdot W_{c s, 0}}{4 G}\left(\frac{\left(t^{\prime}-t_{0}^{\prime}\right)^{3}}{6 t_{0}^{\prime 2}}\right.\right. \\
& \left.\left.-\frac{\left(t^{\prime}-t_{0}^{\prime}\right)^{4}}{8 t_{0}^{\prime 3}}+\cdots\right)\right]
\end{aligned}
$$

For $\left|\left(t^{\prime}-t_{0}^{\prime}\right) / t_{0}^{\prime}\right| \ll 1$, only the first two terms of the expansion are used. In that case, $\phi_{c}\left(t^{\prime}\right)$ results from the integration of Eq. (13) as

$$
\begin{aligned}
\phi_{c}\left(t^{\prime}\right)= & \frac{\pi \cdot W_{c s, 0}}{2 G} \cdot \ln \left(\frac{t_{0}^{\prime}}{t^{\prime}}\right)+\frac{\pi^{3} \cdot W_{c s, 0}^{2}}{288 \cdot G^{2}}\left[6 t_{0}^{\prime} \cdot \ln \left(\frac{t_{0}^{\prime}}{t^{\prime}}\right)\right. \\
& \left.+2 \frac{t^{\prime 3}}{t_{0}^{\prime 2}}-9 \frac{t^{\prime 2}}{t_{0}^{\prime}}+18 t^{\prime}-11 t_{0}^{\prime}\right] .
\end{aligned}
$$

Figure 10 shows three cases with injection energies $W_{s, 0}$ corresponding to 10,30 , and 60 times the gap energy gain $G$.

Now, the resulting gap numbers $N_{l}=\left(t_{f}^{\prime}-t_{0}^{\prime}\right)$ for a transition of the second quadrant in the $W \phi$ plane for different excess energies $W_{c s, 0}$ are calculated.

From Eq. (15) and by using the boundary condition $W_{c s}\left(t_{f}^{\prime}\right)=0$ for the first two terms of the expansion, we get

$N_{l} \cong\left(\frac{24 \cdot W_{s, 0}^{2}}{\pi^{2} \cdot G \cdot W_{c s, 0}}\right)^{1 / 3}=\left[\frac{24}{\pi^{2}} \cdot\left(\frac{W_{s, 0}}{G}\right)^{2} \cdot\left(\frac{W_{c s, 0}}{G}\right)^{-1}\right]^{1 / 3}$.

Figure 11 shows this dependence in a 3D plot. The tolerable number of gaps is quite limited at low injection energies and at high effective gap voltages $V_{\text {eff }}=G / q$. At the same time, high surplus energies $W_{c s, 0}$ also limit the gap number per KONUS section significantly. The frequency does not appear explicitly in Eq. (17). However, the frequency defines the cell length and by this the drift tube geometry, which will reach technical limits at a cell minimum length of currently around $20 \mathrm{~mm}$.

For comparison, three realized KONUS layouts are included in Fig. 11: The black bars indicate gap numbers $N_{l}$ for each zero degree section along the CERN linac3, which accelerates the design particle $\mathrm{Pb}^{25+}$ from $250 \mathrm{~A} \mathrm{keV}$ to $4.2 \mathrm{~A} \mathrm{MeV}[9,10]$. This is a low-current machine with beam currents well below $500 \mu \mathrm{A}$. The frequency is doubled for the second and third cavities with 23 gaps in each KONUS section. The red bars belong to the zero degree sections of the GSI highcurrent injector HSI, which has to deliver $20 \mathrm{~mA}$ of $\mathrm{U}^{4+}$ for the future FAIR facility. The energy range is from $120 \mathrm{~A} \mathrm{keV}$ up to $1.4 \mathrm{~A} \mathrm{MeV}[17,18]$. Finally, the green bars show the FAIR proton linac layout for a $70 \mathrm{~mA}$ beam from 3 to $68 \mathrm{MeV}$ (see Sec. VA and Ref. [24]). This linac is currently under construction. Figure 11 shows that $W_{c s, 0} / G$-the relative surplus in injection
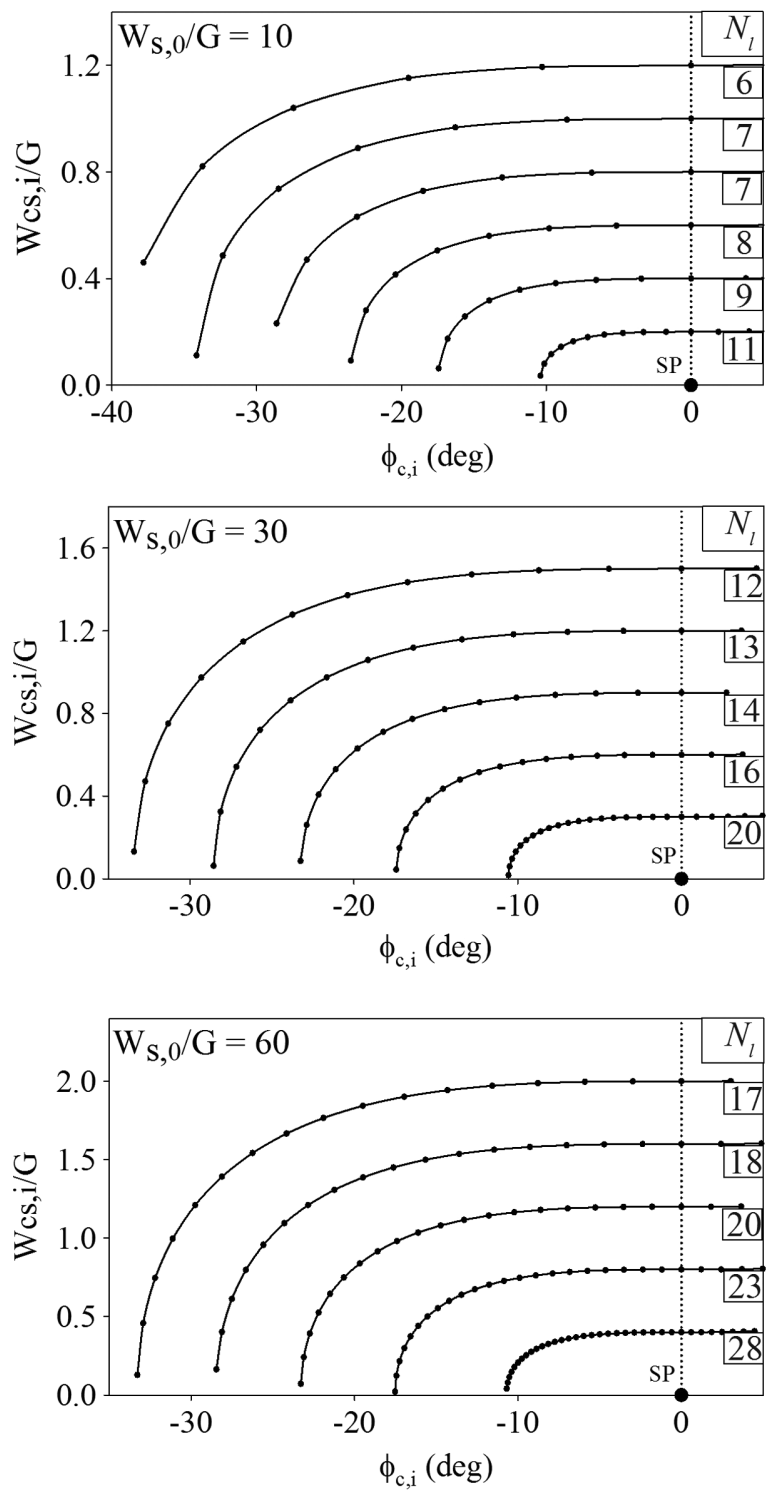

FIG. 10. Traces of the center particle around the synchronous particle for three different ratios of injection energy to gap energy gain $G$ and corresponding gap number values $N_{l}$ in dependence of the surplus in injection energy $W_{c s, 0}$. Dots mark gap centers.

energy -is correlated with the beam current: With a growing space charge load, $W_{c s, 0} / G$ is getting larger at comparable $W_{s, 0} / G$ ratios, resulting in shorter zero degree sections (with lower gap numbers $N_{l}$ and high negative phase increments per cell; see Fig. 10).

\section{B. The single-particle motion}

In a next step, the single-particle motion around the bunch center particle is discussed (see Fig. 12). A comparison will be made between the negative synchronous phase structures (NSPS) and a zero degree KONUS section with respect to the phase advance per period. Moreover, the matched ellipse half axis ratio will be studied. A main 


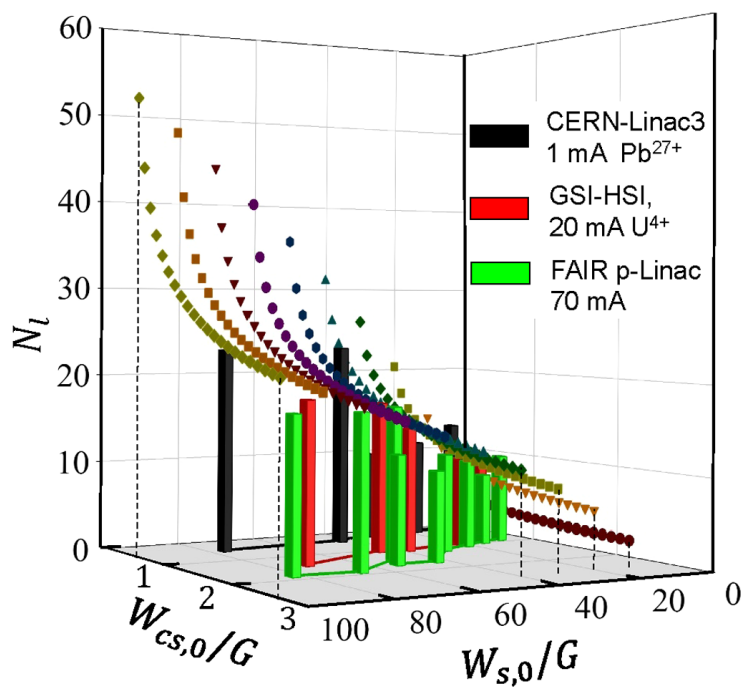

FIG. 11. Illustration of the $N_{l}$ dependence on $W_{s, 0} / G$ and on $W_{c s, 0} / G$. Symbols describe the $N_{l}$ values resulting from Eq. (17) for identical $W_{s, 0} / G$ ratios of $10,20, \ldots 100$, while bars indicate $N_{l}$ values of linac layouts, which are in operation (CERN linac3, GSI high-current injector HSI) or under construction (FAIR p-linac), respectively.

intention is to compare the cyclic motion around the center particle for both cases.

The nonrelativistic equations for a low acceleration rate and for linearized NSPS dynamics (small amplitudes) result with replacing $q \cdot e \cdot E_{0} \cdot T_{T} \cdot \beta_{s} \cdot \lambda$ by $2 G$ in a phase advance per half an rf period [3]:

$$
\sigma_{l}=\left(\frac{-\pi \cdot G \cdot \sin \phi_{s}}{2 \cdot W_{s}}\right)^{1 / 2}
$$

The matched ratio of half axes is

$$
\frac{W_{p c, 0}}{\phi_{p c, 0}}=\left(-\frac{2 G}{\pi} \cdot \sin \phi_{s} \cdot W_{s}\right)^{1 / 2}
$$

$T_{T}$ denotes the gap transit time factor.

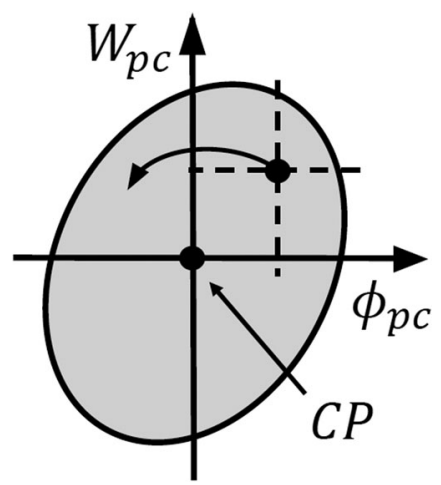

FIG. 12. Scheme of the single-particle motion around the bunch center particle.
The harmonic single-particle motion is described by

$$
\begin{gathered}
\phi_{p c}=\phi_{p c, 0} \cdot \sin \left(\sigma_{l} \cdot t^{\prime}+\alpha\right), \\
W_{p c}=W_{p c, 0} \cdot \cos \left(\sigma_{l} \cdot t^{\prime}+\alpha\right)
\end{gathered}
$$

with the dependencies

$$
\begin{gathered}
\frac{\partial \dot{\phi}_{p c}}{\partial W_{p c}} \cdot \frac{\partial \dot{W}_{p c}}{\partial \phi_{p c}}=-\sigma_{l}^{2}, \\
\frac{\partial \dot{W}_{p c}}{\partial \phi_{p c}} \cdot \frac{\partial W_{p c}}{\partial \dot{\phi}_{p c}}=-\left(\frac{W_{p c, 0}}{\phi_{p c, 0}}\right)^{2} .
\end{gathered}
$$

In the following, corresponding expressions are derived for a zero degree KONUS section, to get some insight into the single-particle motion around the bunch center particle. Using the same procedure as applied to get Eqs. (13) and (14) results in

$$
\begin{gathered}
\dot{W}_{p c}=\frac{G}{2}\left[\phi_{c}^{2}-\left(\phi_{p c}+\phi_{c}\right)^{2}\right], \\
\dot{W}_{p c}=-\frac{G}{2}\left(2 \phi_{p c} \cdot \phi_{c}+\phi_{p c}^{2}\right), \\
\dot{\phi}_{p c}=-\frac{\pi \cdot W_{p c}}{2 \cdot W_{c}} .
\end{gathered}
$$

Insertion of Eqs. (24) and (25) into Eqs. (22) and (23) gives

$$
\frac{\partial \dot{\phi}_{p c}}{\partial W_{p c}} \cdot \frac{\partial \dot{W}_{p c}}{\partial \phi_{p c}}=\frac{\pi \cdot G \cdot\left(\phi_{p c}+\phi_{c}\right)}{2 \cdot W_{c}\left(t^{\prime}\right)}
$$

$$
\frac{\partial \dot{W}_{p c}}{\partial \phi_{p c}} \cdot \frac{\partial W_{p c}}{\partial \dot{\phi}_{p c}}=\frac{2 \cdot G \cdot W_{c}\left(t^{\prime}\right)}{\pi} \cdot\left(\phi_{p c}+\phi_{c}\right) .
$$

Averaging along a zero degree KONUS section from $t_{0}^{\prime}$ to $t_{f}^{\prime}$ and summing over all bunch particles results in a squared phase advance per $\mathrm{rf}$ half period of

$$
\left\langle\sigma_{l}^{2}\right\rangle=-\frac{\pi \cdot G}{2 \cdot N} \cdot \sum_{j=1}^{f} \frac{\phi_{c, j}}{W_{c, j}}
$$

and in a squared half axis ratio of

$$
\left\langle\left(\frac{W_{p c, 0}}{\phi_{p c, 0}}\right)^{2}\right\rangle=-\frac{2 \cdot G}{\pi \cdot N} \cdot \sum_{j=i}^{f} \phi_{c, j} \cdot W_{c, j} .
$$

One can clearly see the necessity of mainly negative phases $\phi_{c}$ to get a stable beam dynamics. As explained above and illustrated by Fig. 4 , the trace $\phi_{c}\left(t^{\prime}\right)$ is defined by its starting point relative to the synchronous zero degree 
particle which is used to define the drift tube array. If the bunch center particle is injected at phases around zero degree but with a surplus energy $W_{c s, 0}$ like shown in Figs. 6 and 8, all bunch particles are moving towards negative phases, and this allows the use of acceleration rf phases rather close to the crest of the wave along the first few gaps of each zero degree KONUS section. Moreover, both equations depend linearly on the effective gap voltage amplitude $G / q$.

A zero degree KONUS section design has as its main parameters the gap number $N_{l}$, the effective gap voltage $G / q$, and the surplus energy $W_{c s, 0}=W_{c s}\left(t_{0}^{\prime}\right)$. The design parameter $W_{c s, 0}$ has to be readjusted accordingly, when beam design parameters like emittance or current are substantially increased (compare Fig. 11 for realized cases). However, the operation of an "overdesigned" KONUS structure at a reduced beam current or for ions lighter than the design particle will be of no issue, as long as the longitudinal phase advance does not become too large. This results from beam operation experience and from numerous beam simulations [2].

Another reason for limiting $N_{l}$ to even smaller numbers than allowed by longitudinal beam dynamics is the transverse beam dynamics, which will be discussed in the next section.

\section{TRANSVERSE BEAM DYNAMICS ALONG A KONUS PERIOD}

\section{A. Length limitation of the acceleration section}

At the DTL front end, the tolerable gap number per KONUS section is in many cases even more restricted by the transverse beam dynamics. At a given beam emittance and drift tube aperture radius $a$, a maximum gap number $N_{\text {tot }}$ can be estimated due to transverse phase-space restrictions and by applying the following assumptions: (i) The beam radius is $a / 4$ at the beam waist, (ii) particles with maximum transverse momenta are located at $r=a / 2$ at the injection and exit of the drift tube section, (iii) beam acceleration and $\mathrm{rf}$ defocusing result in approximately unchanged $x^{\prime}$ and $y^{\prime}$ at the section exit, and (iv) no space charge action is involved.

The total acceleration length $L$ (see Fig. 2) is given by

$$
L=\int_{0}^{N_{\mathrm{tot}} \cdot T / 2} v(t) \cdot d t
$$

with

$$
v(t)=\left(\frac{2 \cdot W_{s, 0}}{m}+\frac{4 \cdot G \cdot t}{T \cdot m}\right)^{1 / 2},
$$

resulting in

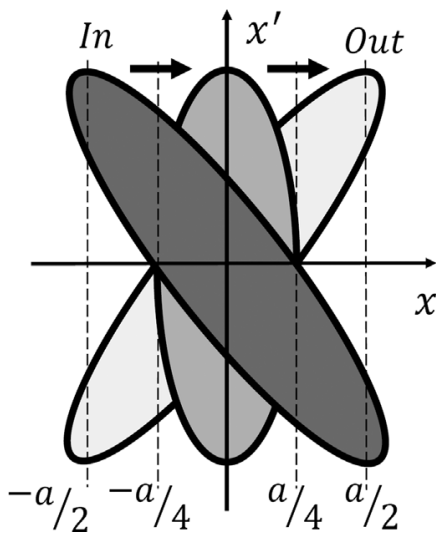

FIG. 13. Transverse ellipse transformation along one KONUS drift tube section.

$L=\frac{m}{6 \cdot G \cdot f} \cdot\left(\frac{2}{m}\right)^{3 / 2}\left[\left(N_{\mathrm{tot}} \cdot G+W_{s, 0}\right)^{3 / 2}-W_{s, 0}^{3 / 2}\right]$

with the operating frequency $f=T^{-1}$. A given effective beam ellipse with emittance $\varepsilon$ is changed along the KONUS section approximately according to Fig. 13. The maximum transverse angle $x_{\max }^{\prime}$ of a particle with respect to the beam axis is given by

$$
x_{\max }^{\prime}=\frac{4 \varepsilon}{a},
$$

and geometrical conditions result in

$$
L=\frac{a}{x_{\max }^{\prime}}
$$

Inserting $L$ from Eq. (34) into Eq. (32) gives an upper gap number limit $N_{\text {tot }}$ caused by the transverse beam dynamics:

$N_{\mathrm{tot}}=\left[\left(\frac{W_{s, 0}}{G}\right)^{3 / 2}+\frac{3 a^{2} \cdot f}{4 \cdot \varepsilon} \cdot\left(\frac{m}{2 G}\right)^{1 / 2}\right]^{2 / 3}-\frac{W_{s, 0}}{G}$.

As shown by Fig. 2, $N_{\text {tot }}$ has to include the rebunching gaps in front of the zero degree section, while $N_{l}$ was defined in Sec. III A by taking into account the zero degree section only.

The maximum tolerable gap number $N$ per KONUS section is then estimated by

$$
N=\min \left[\left(N_{l}+N_{\text {reb }}\right), N_{\text {tot }}\right] .
$$

In the following, the transverse and longitudinal beam dynamics for one KONUS period is investigated in more detail by the matrix multiplication method, and stable parameter ranges are derived. 
The special quadrupole triplet design in KONUS results from the necessity to have as short lenses as possible in order to keep the bunch length short. The result are high magnetic field gradients and minimized distances between singlets.

\section{B. Quadrupole triplet layout}

At first, the features of compact high field triplets are estimated. An explicit calculation along a triplet lens consisting of two outer singlets with length $l$ and an inner singlet with length $2 l$ and at identical field gradients was performed. The drifts between effective singlet field lengths are set to zero; this is a good approximation to the designs used for KONUS structures. A scheme of a compact KONUS intertank section is shown in Fig. 14.

The quadrupole constant $k$ (see, e.g., Ref. [3]) is written as

$$
k=\left|\left(\frac{q \cdot B^{\prime}}{m c \beta \gamma}\right)^{1 / 2}\right| ; \quad \alpha=k \cdot l ;
$$

$B^{\prime}$ denotes the magnetic field gradient, and $l$ is the effective magnetic length.

The focusing quadrupole transfer matrix of a focusing outer singlet reads as

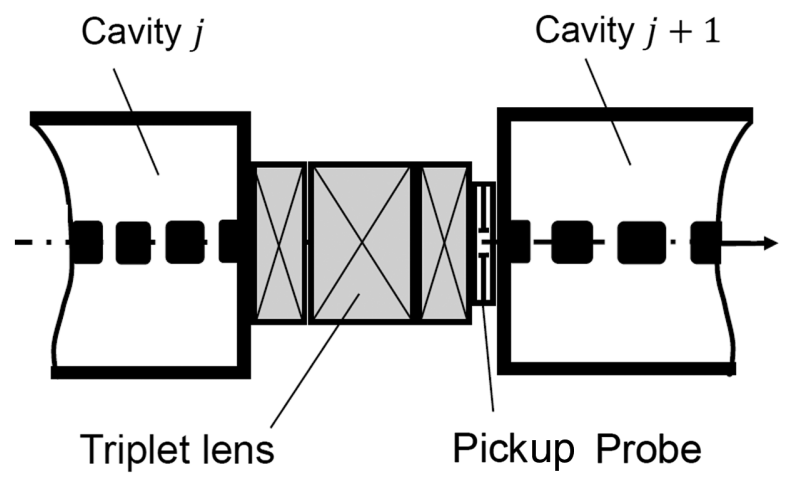

FIG. 14. Sectional view of an intertank section consisting of a bunch pickup probe for beam diagnostics and of a compact quadrupole triplet. The length between neighbored cavities must be minimized for an optimum longitudinal beam transport.

$$
Q_{F}=\left(\begin{array}{cc}
\cos \alpha & \frac{\sin \alpha}{k} \\
-k \sin \alpha & \cos \alpha
\end{array}\right)
$$

For a defocusing quadrupole transfer matrix of a defocusing inner singlet, one gets

$$
Q_{D}=\left(\begin{array}{cc}
\cosh 2 \alpha & \frac{\sinh 2 \alpha}{k} \\
k \sinh 2 \alpha & \cosh 2 \alpha
\end{array}\right) \text {. }
$$

By multiplication, one gets the matrix for the FDF case

$$
Q_{\mathrm{FDF}}=\left(\begin{array}{cc}
\cosh 2 \alpha \cdot \cos 2 \alpha & (\cosh 2 \alpha \cdot \sin 2 \alpha+\sinh 2 \alpha) / k \\
(\sinh 2 \alpha-\cosh 2 \alpha \cdot \sin 2 \alpha) \cdot k & \cosh 2 \alpha \cdot \cos 2 \alpha
\end{array}\right)
$$

and for the DFD case

$$
Q_{\mathrm{FDF}}=\left(\begin{array}{cc}
\cosh 2 \alpha \cdot \cos 2 \alpha & (\sinh 2 \alpha \cdot \cos 2 \alpha+\sin 2 \alpha) / k \\
(\sinh 2 \alpha \cdot \cos 2 \alpha-\sin 2 \alpha) \cdot k & \cosh 2 \alpha \cdot \cos 2 \alpha
\end{array}\right) .
$$

In Taylor expansion, the trigonometric functions are replaced by

$$
\begin{array}{rlrl}
\sin 2 \alpha & \cong 2 \alpha-\frac{4}{3} \alpha^{3} ; & \cos 2 \alpha \cong 1-2 \alpha^{2} ; \\
\sinh 2 \alpha \cong 2 \alpha+\frac{4}{3} \alpha^{3} ; & \cosh 2 \alpha \cong 1-2 \alpha^{2} .
\end{array}
$$

This results in approximated expressions for small $\alpha$

$Q_{\mathrm{FDF}} \cong\left(\begin{array}{cc}1-4 k^{4} l^{4} & 4 l+4 k^{2} l^{3}-\frac{8}{3} k^{4} l^{5} \\ -\frac{4}{3} k^{4} l^{3}+\frac{8}{3} k^{6} l^{5} & 1-4 k^{4} l^{4}\end{array}\right)$

and

$$
Q_{\mathrm{DFD}} \cong\left(\begin{array}{cc}
1-4 k^{4} l^{4} & 4 l-4 k^{2} l^{3}-\frac{8}{3} k^{4} l^{5} \\
-\frac{4}{3} k^{4} l^{3}-\frac{8}{3} k^{6} l^{5} & 1-4 k^{4} l^{4}
\end{array}\right)
$$

The matrix elements $Q_{21}$ represent the resulting focusing strength of the triplet. The leading terms are identical in both planes, while the second term changes sign. It means that the focal length of the DFD plane is shorter. This effect is clearly seen in KONUS designs. As a consequence, the central triplet is significantly shorter than $2 l_{1}$ at all lowenergy sections. With $l_{2}=(1-\delta) l_{1}$ as defined in Fig. 15, a typical design value at beam energies below $2 \mathrm{~A} \mathrm{MeV}$ is $\delta=0.12$. By that, the field gradients are very similar for all singlets. Technically, the first and third singlets are operated in series, while the central one has its own power supply. 


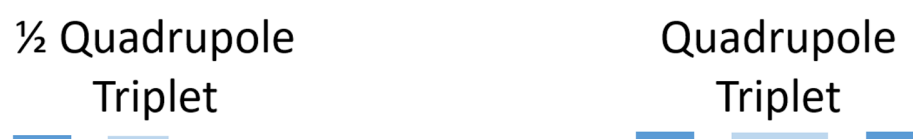

\section{$1 / 2$ Quadrupole Triplet}

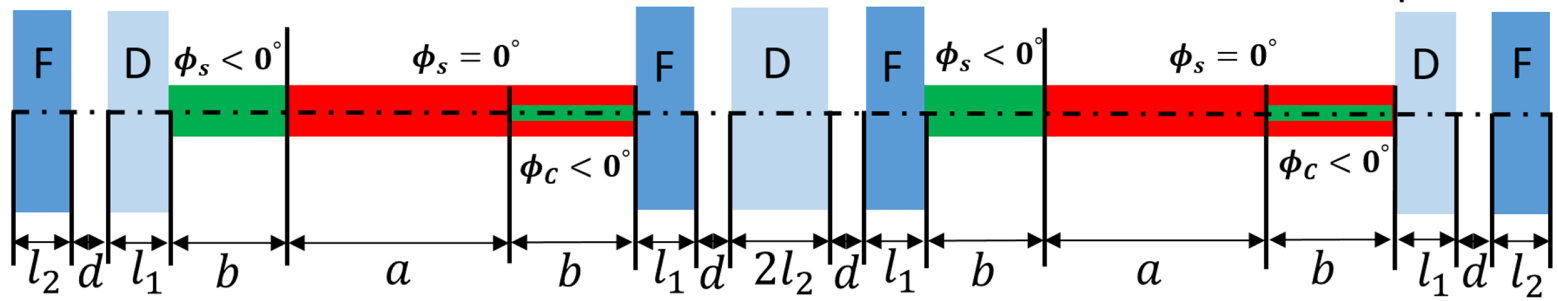

FIG. 15. Scheme of a KONUS period in a DFD-O-FDF-O- focusing lattice. The red bar marks the extension of the zero degree section.

The FDF-O-DFD-O lattice was proven by numerous beam simulations to be best suited for KONUS. In this case, each KONUS period contains two triplets. The transversal design of such a KONUS period using the matrix formalism is shown in the following subsection.

\section{Transverse transfer matrix and stability chart}

One KONUS period can be described in more detail by the following parameters: (i) quadrupole lengths $l_{1}$ and $2 l_{2}$, (ii) drift between the quadrupoles $d$, and (iii) bunch phase $\phi_{c}$ and effective voltage $V_{i}$ for each gap.

Additionally, the energy, mass, and charge of the accelerated particles are needed to calculate the corresponding period length and the focusing or defocusing action of quadrupoles and gaps.

Figure 15 presents a scheme with geometrical parameters for a DFD-O-FDF-O- periodical KONUS lattice.

The transversal focusing or defocusing by one gap can be represented by a thin-gap approximation, which is a lens with inverse focal length $s$. The gap matrix for a $\beta \lambda / 2$ structure with cell-averaged acceleration field amplitude $E_{0}$ reads as follows:

$$
G=\left(\begin{array}{ll}
1 & 0 \\
s & 1
\end{array}\right) \text { with } \quad s=\frac{-\pi q E_{0} T_{T} \sin \phi}{2 m c^{2} \gamma^{3} \beta^{2}} .
$$

The expression for $s$ is derived, e.g., in Ref. [3] for a $\beta \lambda$ structure, resulting accordingly in an extra factor of 2. $T_{T}$ denotes the gap transit time factor. For each acceleration section, one will have to calculate each gap by itself, which will result in the following calculation for the full transfer matrix, where $P$, at negligible energy gain, represents the matrix for a constant drift $p=\beta \lambda / 2$ :

$$
G_{1 \rightarrow n}=P \cdot G_{n} \cdot P \cdot G_{n-1} \cdots \cdot P \cdot G_{2} \cdot P \cdot G_{1}=\prod_{i=1}^{n} P \cdot G_{i} .
$$

For an exact result, this procedure is necessary, but for a first estimation and in the case of a huge number of gaps, it is reasonable to sum up all gaps in one transfer matrix. For this, the acceleration section is divided into the following unit cells:

$U=\left(\begin{array}{ll}1 & \frac{p}{2} \\ 0 & 1\end{array}\right)\left(\begin{array}{ll}1 & 0 \\ s & 1\end{array}\right)\left(\begin{array}{ll}1 & \frac{p}{2} \\ 0 & 1\end{array}\right)=\left(\begin{array}{cc}1+\frac{s p}{2} & p+\frac{s p^{2}}{4} \\ s & 1+\frac{s p}{2}\end{array}\right)$.

The full transfer matrix for an accelerating section containing $n$ gaps is therefore the following:

$$
U^{n}=\left(\begin{array}{cc}
1+s p \cdot 0.5 n^{2}+(s p)^{2} \cdot \tau+\cdots & n p+s p^{2} \sigma_{2}+\cdots \\
n s+s^{2} p \sigma_{1}+\cdots & 1+s p \cdot 0.5 n^{2}+(s p)^{2} \cdot \tau+\cdots
\end{array}\right)
$$

with

$$
\begin{aligned}
\tau & =\frac{1}{24}\left(\tilde{n}^{4}+4 \tilde{n}^{3}+5 \tilde{n}^{2}+2 \tilde{n}\right) ; \quad \tilde{n}=n-1, \\
\sigma_{1} & =\frac{1}{6} \tilde{n}^{3}+\frac{1}{2} \tilde{n}^{2}+\frac{1}{3} \tilde{n} ; \quad \sigma_{2}=\frac{1}{4} n+\sigma_{1} .
\end{aligned}
$$

As usual, for the transverse beam dynamics investigation, the acceleration itself is now neglected. Along the rebunching section with length $b$ and gap number $n$, the synchronous phase $\phi_{s}$ is applied in Eq. (46). The transversally defocusing action of the following zero degree section is located mainly at its last gaps and is approximated by a drift $a$ followed by a rebunching section which is identical with the rebunching section at the front end, again with length $b$ (see Fig. 15). This way of simulating the contribution from the zero degree section has proven to be a good approximation.

With this simplification, one can derive the following calculation for one KONUS period ( $D$ and $A$ denote the drift matrices along $d$ and $a$, respectively; see Fig. 15): 


$$
T=\underbrace{Q_{F l_{2}} \cdot D \cdot Q_{D l_{1}}}_{\frac{1}{2} \text { Quadrupole Triplet }} \cdot \overbrace{U^{n} \cdot A \cdot U^{n}}^{\text {Acceleration Section }} \cdot \underbrace{Q_{F l_{1}} \cdot D \cdot Q_{D l_{2}}^{2} \cdot D \cdot Q_{F l_{1}}}_{\text {Quadrupole Triplet }} \cdot \overbrace{U^{n} \cdot A \cdot U^{n}}^{\text {Acceleration Section }} \cdot \underbrace{Q_{D l_{1}} \cdot D \cdot Q_{F l_{2}}}_{\frac{1}{2} \text { Quadrupole Triplet }} \cdot
$$

According to Ref. [3], one can derive the phase advance $\mu$ per structure period from the corresponding transfer matrix $T$, by using the following abstract representation between two points with identical Twiss parameters:

$$
T=\left(\begin{array}{cc}
\cos \mu+\alpha \sin \mu & \beta \sin \mu \\
-\gamma \sin \mu & \cos \mu-\alpha \sin \mu
\end{array}\right) .
$$

For a stable beam motion, this matrix has to be limited. This requirement will be fulfilled if the absolute value of the trace is smaller than 2 :

$$
|\operatorname{Tr}(T)|=2|\cos \mu| \leq 2 .
$$

For a fixed energy, charge, and mass, one can calculate for different structure parameters $s$ and $k$ the corresponding phase advance in the defined structure period. Because of the limitation of $\cos \mu$ to the interval $[-1,1]$, a stable beam motion is possible only for a certain space in the $s k$ plane.

While a period containing one lens is limited to a maximum of $180^{\circ}$ phase advance per period, in the "two triplets per period" case, a phase advance up to $360^{\circ}$ has to be expected. The matrix formalism is conformed with that extension by applying the following rule: If $T$ is the transfer matrix for an arbitrary structure containing triplets, one has to examine two cases regarding the sign of the matrix elements $t_{12}$ and $t_{21}$ of the matrix $T$ :

$$
\begin{gathered}
\text { If } t_{21}<0 \text { and } t_{12}>0, \\
\text { it follows } \mu=\arccos \left(\frac{1}{2}|\operatorname{Tr}(T)|\right) ; \\
\text { if } t_{12}<0 \text { and } t_{21}>0, \\
\text { it follows } \mu=2 \pi-\arccos \left(\frac{1}{2}|\operatorname{Tr}(T)|\right) .
\end{gathered}
$$

This results in the formation of two separated areas of stable beam motion in the $s k$ plane and, therefore, a discontinuity for phase advances at $180^{\circ}$, as shown for one parameter set in Fig. 17.

The discontinuity can be locally minimized around one given parameter value $s$ (blue vertical line in Fig. 17) through optimizing the ratio between the quadrupole lengths $l_{1}$ and $l_{2}$, which is smaller than one as mentioned above. Equivalently, one can vary the field ratio between the outer and the center quadrupoles [1]. The instability around $180^{\circ}$ can be intuitively characterized by Fig. 16 .
The matched solution shows a sudden change from envelope type 1 to type 2, while the phase advance keeps very close to $180^{\circ}$.

Additionally, the ratio of the maximum and minimum transverse beam widths along a period, the so-called flutter factor [3], is plotted in Fig. 17.

For quadrupole triplet channels with FDF-O-DFD-O periodicity, the following definition for the flutter factor $\Psi$ is introduced:

$$
\Psi=\left[\frac{\left(\beta_{F} \cdot \beta_{D}\right)^{1 / 2}}{\beta_{\text {waist }}}\right]^{1 / 2} .
$$

$\beta_{F}$ and $\beta_{D}$ denote the maxima of the beta functions along the FDF and DFD triplet paths, respectively. $\beta_{\text {waist }}$ denotes the minimum beta value at the waist within the acceleration section. These three characteristic $\beta$ values are derived via the matrix element $t_{12}$ at a given phase advance $\mu$. The redefinition of the starting point $z$ of the structure period results in the calculation of $\beta(z)$.

The set of parameters used for Fig. 17 for the case of proton acceleration is

$$
\begin{aligned}
& E_{0} \cdot T_{T} \cdot \sin \phi_{s}=-2.3 \mathrm{MV} / m ; \quad \overline{W_{s}}=33 \mathrm{MeV} \\
& l_{1}=52 \mathrm{~mm} ; \quad l_{2}=49.8 \mathrm{~mm} ; \quad d=18 \mathrm{~mm} ; \\
& a=1.428 \mathrm{~m} \equiv 12 \frac{\beta \lambda}{2} ; \quad b=0.476 \mathrm{~m} \equiv 4 \frac{\beta \lambda}{2} \\
& n=4 ; \quad \phi_{s}=-35^{\circ} .
\end{aligned}
$$

From according particle simulations, it is deduced that these parameters describe a case in which an energy gain of $11 \mathrm{MeV}$ is achieved at $325 \mathrm{MHz}$ and within a cavity length of $2.4 \mathrm{~m}$ for protons at a remarkable current limit of around $100 \mathrm{~mA}$ (see Sec. V A, proton linac design).

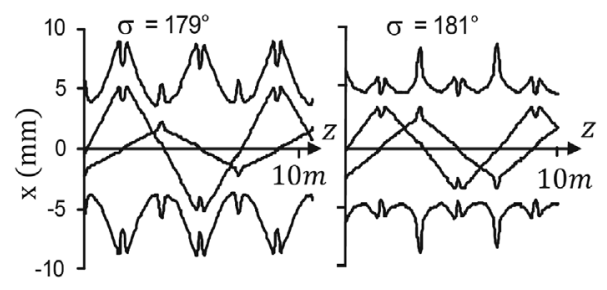

FIG. 16. Plot of the envelope flip for two matched solutions around $\sigma=180^{\circ}$. 


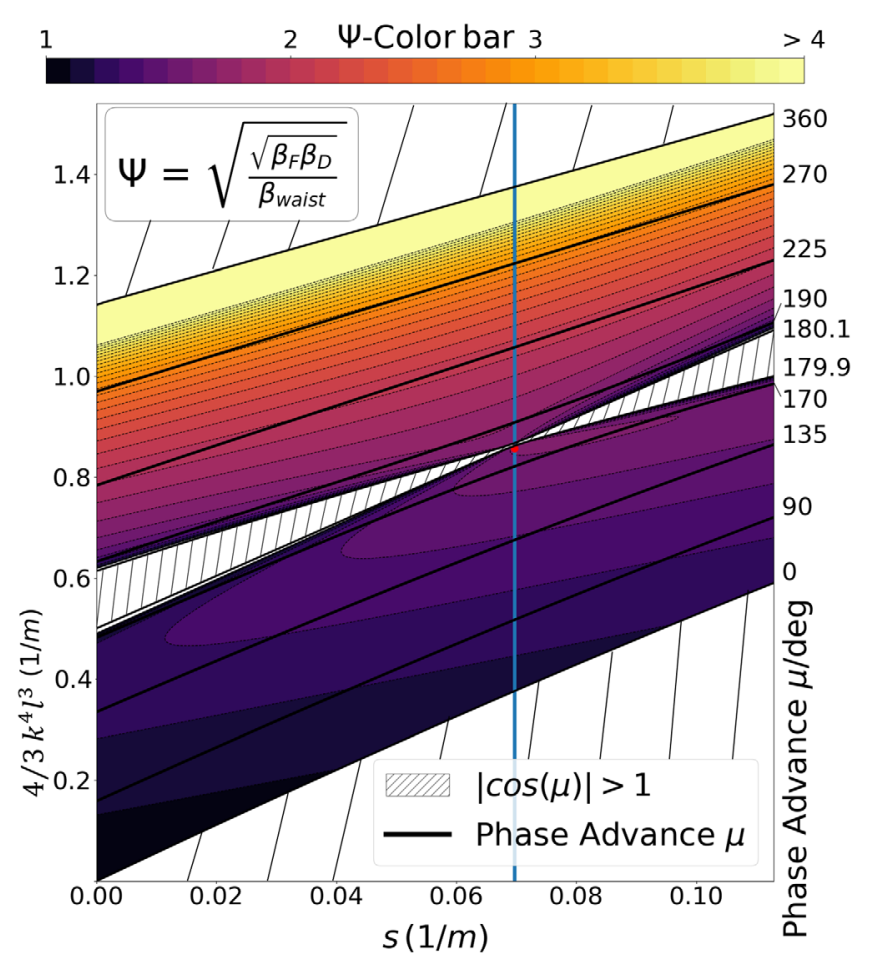

FIG. 17. Stability chart for the transverse motion in a KONUS structure with a FDF-O-DFD-O lattice: The abscissa denotes the inverse focal length of the defocusing gaps, while the ordinate displays the leading term [see Eqs. (43) and (44)] of the triplet inverse focal length. (The plot corresponds to the stability chart invented for FODO structures by Smith and Gluckstern [25].) The blue line marks the $s$ value for minimized beam instability at a transverse phase advance around $180^{\circ}$. The colors indicate the distribution of the flutter factor.

\section{BEAM DYNAMICS SIMULATION RESULTS}

In the following, two KONUS design cases will be described in detail. In case A, we will focus on the longitudinal beam motion and the KONUS lattice. In case $\mathrm{B}$, the transverse beam motion will be showcased. Case B additionally contains a parameter error analysis. KONUSdrift tube arrays are designed with the code LORASR, which was specifically developed for this purpose (see the Appendix and Ref. [26]), while multiparticle simulations
TABLE I. Beam input parameters of the $65 \mathrm{MV}$ proton linac.

\begin{tabular}{lc}
\hline \hline Ion species & Proton \\
Beam current & $70 \mathrm{~mA}$ \\
Transv. emittance $\varepsilon_{n, \mathrm{rms}}$ & $0.3 \mathrm{~mm} \mathrm{mrad}$ \\
Long. emittance $\varepsilon_{\mathrm{rms}}$ & $2.39 \mathrm{keV} \mathrm{ns}$ \\
Injection energy & $3 \mathrm{MeV}$ \\
\hline \hline
\end{tabular}

and error studies on a given drift tube array can be performed by many existing linac codes. The simulations presented in this section are performed with LORASR and with TRACEWIN [27].

\section{A. High current proton acceleration of up to $68 \mathrm{MeV}$}

During the past two decades, a multigap layout of $\mathrm{H}$-mode cavities has been developed which is suitable for a wide velocity range, as is needed for proton acceleration. The IH structure $\left(H_{11(0)}\right.$ mode) is efficient up to around $30 \mathrm{~A} \mathrm{MeV}$. It is providing convenient transverse dimensions for manufacturing cavities with frequencies below $300 \mathrm{MHz}$ in the traditional way. On the other hand, the $\mathrm{CH}$ structure $\left(H_{21(0)}\right.$ mode) is efficient up to around $100 \mathrm{~A} \mathrm{MeV}$ and allows one to realize cavities with operating frequencies ranging from 200 to $600 \mathrm{MHz}$, which is quite suitable for proton acceleration.

The following example shows a high-current $68 \mathrm{MeV}$ proton linac based on CH-type cavities [28]. A novel ladder RFQ [29] provides $3 \mathrm{MeV}$ protons to the entrance of the CH-type linac.

In order to reach the desired energy of $68 \mathrm{MeV}$, a total acceleration of $65 \mathrm{MV}$ is required. The effective voltage gain of $65 \mathrm{MV}$ is applied by six CH-type cavities within a total accelerator length of $21 \mathrm{~m}$ (see Fig. 18). The first three cavities are so-called "coupled-CH" structures, which include an internal quadrupole triplet lens: The two KONUS sections of these $\mathrm{CCH}$ cavities are rf coupled by the coupling cell containing the triplet. This allows for the construction of six cavities which can economically be fed by six identical power klystrons. In total, 12 quadrupole triplets are needed for transverse focusing along the linacincluding two transport sections at 3 and $33 \mathrm{MeV}$. The beam input parameters for this example are shown in Table I. A 6D

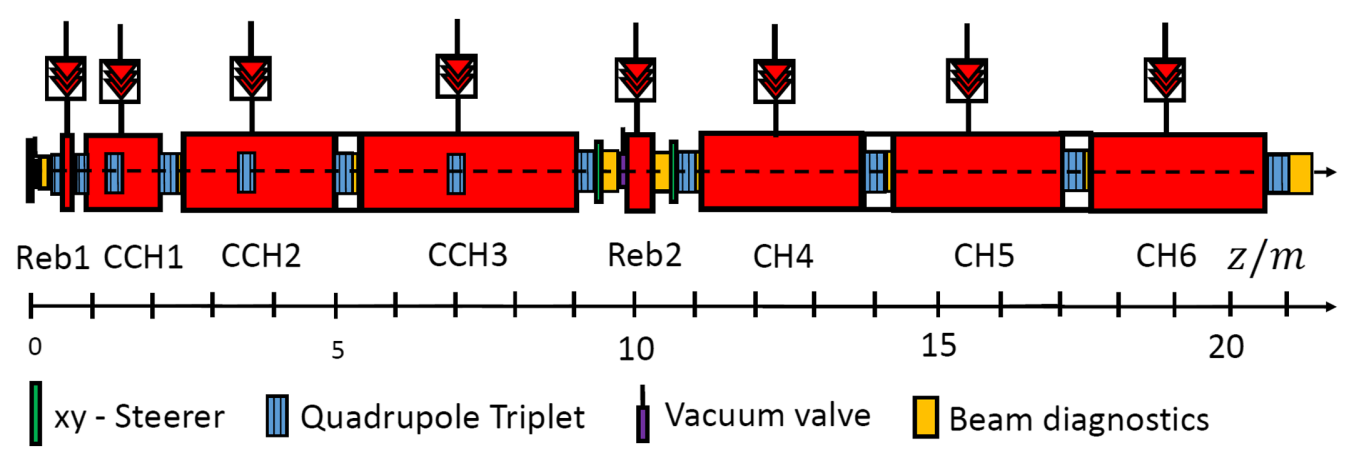

FIG. 18. Scheme of the $65 \mathrm{MV}$ GSI/FAIR proton DTL with main components. 
water bag distribution was used for the following simulations. The effective voltage distributions and maximum onaxis $\mathrm{E}$ fields are shown in Fig. 19. In $\mathrm{CCH} 3$, the overall voltage is reduced in order to stay within given power limits. The main linac parameters are summarized in Table II.

Each of the three $\mathrm{CCH}$ cavities features two KONUS periods, one in front of the focusing lens and one behind the lens. However, in the $\mathrm{CCH} 1$ cavity, the first rebuncher section is omitted, since the longitudinal focusing and matching is provided by the buncher in the medium energy beam transport section. All rebuncher sections have a synchronous phase of $\varphi_{s}=-35^{\circ}$. The longitudinal motion of the bunch center for each zero degree section is shown in Fig. 20. The zero degree sections of the linac are designed with surplus energies of $2 \%-10 \%$ and starting phases around $0^{\circ}$.

The highest surplus energies are needed for the low- $\beta$ section of the linac. The surplus energy is reduced gradually with increasing particle velocities. Figure 21 shows the relative energy and phase envelopes in reference to the structure synchronous particle which illustrates the KONUS periods. The stability of the longitudinal beam motion is apparent from Fig. 22, which shows the beam envelopes relative to the bunch center.
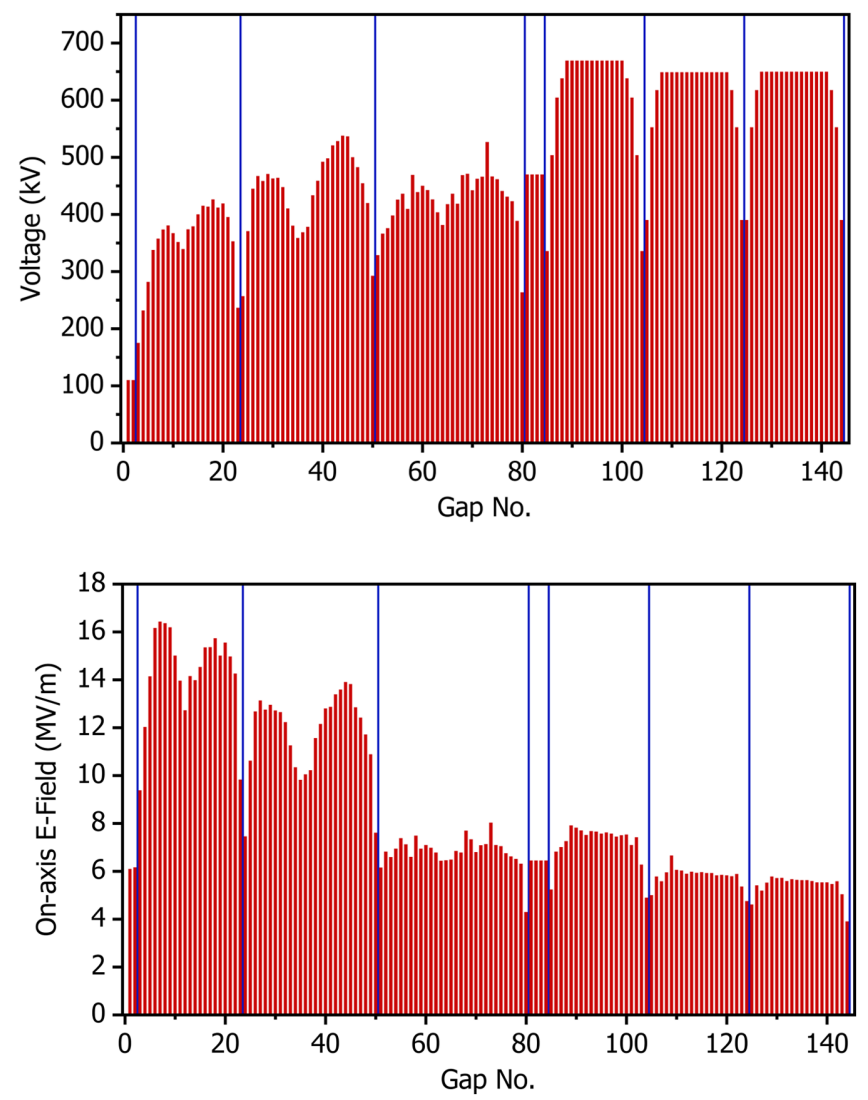

FIG. 19. Gap voltage (top) and on-axis maximum gap field distribution along the CH-DTL and including the $33 \mathrm{MeV}$ rebuncher.
TABLE II. 65 MV DTL parameters.
Total length

Operating frequency

Avg. acc. gradient

Acc. cavities

Rebunchers

Quadrupole triplets

Max on-axis field

Magnetic gradients

KONUS sections

Gaps per section
$21 \mathrm{~m}$

$325.224 \mathrm{MHz}$

$3.1 \mathrm{MV} \mathrm{m}^{-1}$

6

2

12

$8-16 \mathrm{MV} \mathrm{m}^{-1}$

$8-68 \mathrm{~T} \mathrm{~m}^{-1}$

9 in total

8 rebunching

9 zero degree

3-5 (rebunching)

8-17 (zero degree)
The output beam parameters of this KONUS proton linac design are summarized in Table III. For injection into the synchrotron, the energy spread of the beam has to be as small as possible. Therefore, a debuncher cavity is used after the beam has drifted a longer section of the transfer

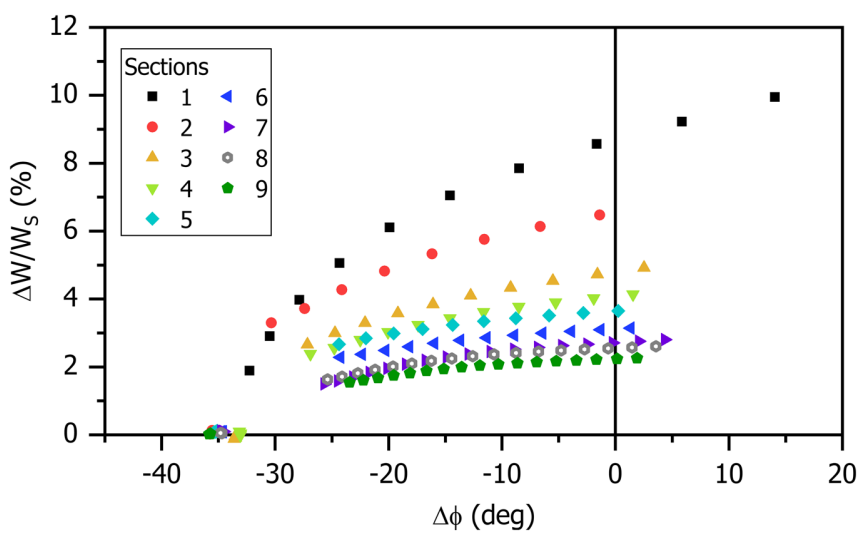

FIG. 20. Bunch center motion along the whole $65 \mathrm{MV}$ proton linac.

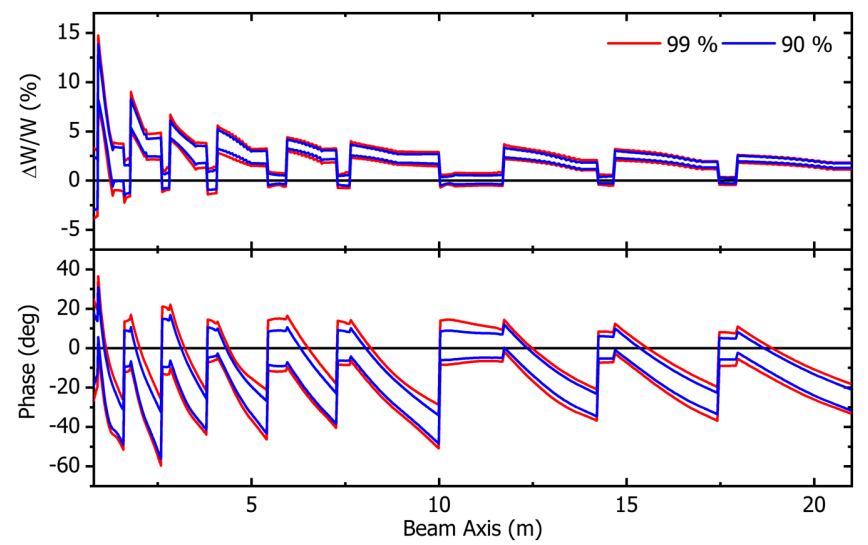

FIG. 21. Longitudinal beam envelopes along the $65 \mathrm{MV}$ proton DTL. The envelopes are shown relative to the synchronous particle to illustrate the KONUS beam dynamics. 


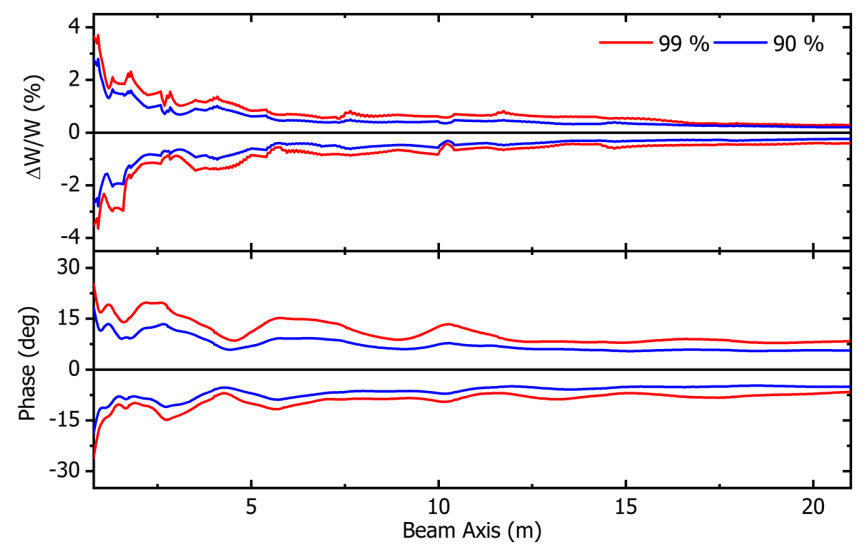

FIG. 22. Longitudinal beam envelopes along the $65 \mathrm{MV}$ proton DTL, with envelopes centered around the bunch center particle.
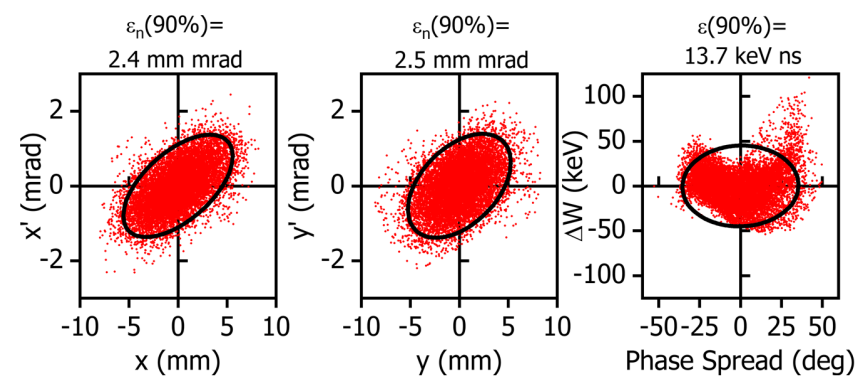

FIG. 23. Transverse and longitudinal particle distributions at $68 \mathrm{MeV}$ behind a $15 \mathrm{~m}$ drift and a six-gap debuncher.

line. An example of this linac's exit emittances and energy spread is shown in Fig. 23. In this case, the beam was transported $15 \mathrm{~m}$ behind the linac exit, passing a six-gap debuncher at the end. The resulting energy spread $(90 \%$ of particles) is $\pm 45 \mathrm{keV}$, which corresponds to $\pm 0.7 \times 10^{-3}$ of the final linac energy and fulfills the synchrotron requirements quite well.
TABLE III. Beam output parameters of the $65 \mathrm{MV}$ proton linac.

\begin{tabular}{lc}
\hline \hline Beam current & $70 \mathrm{~mA}$ \\
Transmission & $100 \%$ \\
Trans. emittance $\varepsilon_{n, \mathrm{rms}}$ & $0.53 \mathrm{~mm} \mathrm{mrad}$ \\
Long. emittance $\varepsilon_{\mathrm{rms}}$ & $2.56 \mathrm{keV} \mathrm{ns}$ \\
Exit energy & $68 \mathrm{MeV}$ \\
\hline \hline
\end{tabular}

TABLE IV. Beam input parameters of the $85 \mathrm{MV}$ heavy ion linac.

\begin{tabular}{lc}
\hline \hline Ion species & $\mathrm{U}^{28+}$ \\
Mass over charge ratio & $\mathrm{A} / \mathrm{q}=8.5$ \\
Macropulse current & $15 \mathrm{~mA}$ \\
Trans. emittance $\varepsilon_{n, \mathrm{rms}}$ & $0.19 \mathrm{~mm} \mathrm{mrad}$ \\
Long. emittance $\varepsilon_{\mathrm{rms}}$ & $1.55 \mathrm{~A} \mathrm{keV} \mathrm{ns}$ \\
Injection energy & $1.4 \mathrm{~A} \mathrm{MeV}$ \\
\hline \hline
\end{tabular}

\section{B. High-current heavy ion acceleration up to 11.4 A MeV}

As described in the introduction already, the KONUS development has been driven by heavy ion accelerator projects. In the following case [30], we take a high-current heavy ion beam example at the cutting edge of present ion source and preaccelerator technology. The main input parameters are listed in Table IV. For the following simulations and error studies, a 6D water bag distribution has been used as the input distribution.

In this example, a $\mathrm{U}^{28+}$ beam is accelerated from 1.4 to 11.4 A MeV for a high beam current of $15 \mathrm{~mA}$. The required effective voltage gain of $85 \mathrm{MV}$ is applied by five IH-type cavities within a total accelerator length of $23 \mathrm{~m}$. A total of seven quadrupole triplets is needed for transverse focusing (see the layout in Fig. 24). The first cavity features two internal quadrupole triplet lenses, in order to ensure adequate transverse focusing at a low beam velocity. The remaining

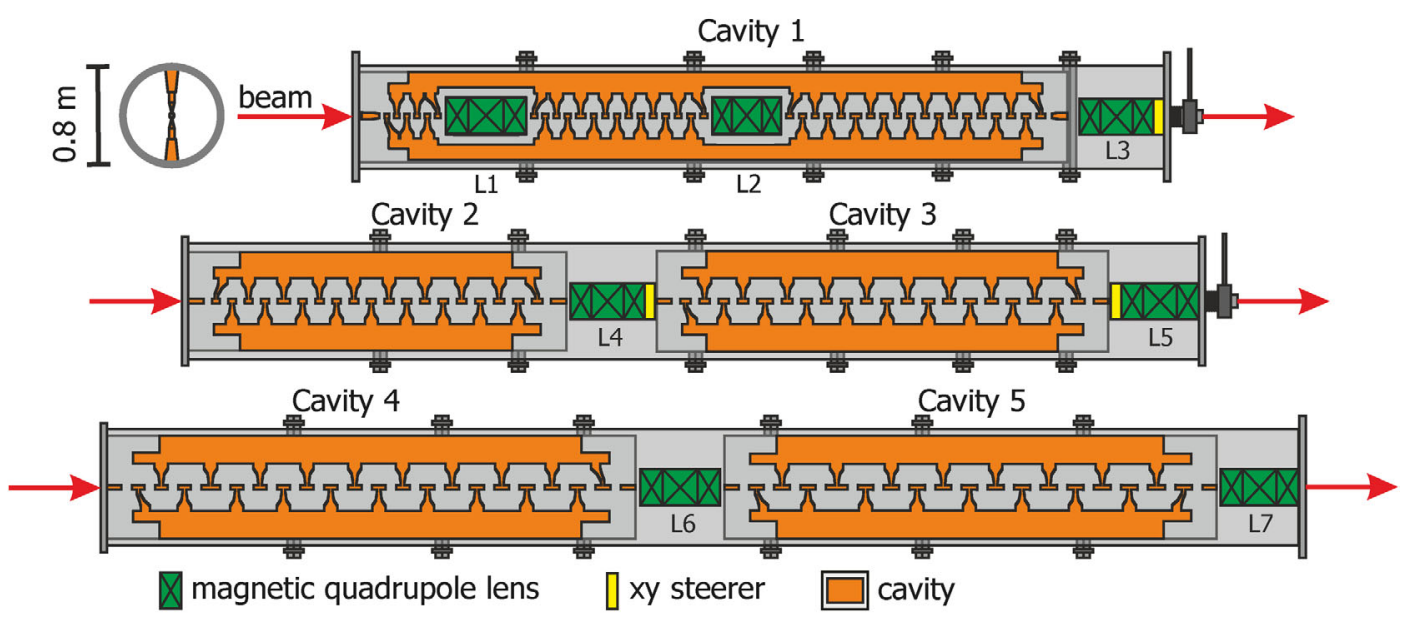

FIG. 24. Layout of the five IH-DTL cavities comprising three separate rigid tanks. 
TABLE V. 85 MV linac parameters.

Total length

Operating frequency

Avg. acc. gradient

Cavities

Quadrupole triplets

Max on-axis field

Magnetic gradients

KONUS sections

Gaps per section
$22.8 \mathrm{~m}$

$108.408 \mathrm{MHz}$

$3.7 \mathrm{MV} \mathrm{m}^{-1}$

5

7

$5-11 \mathrm{MV} \mathrm{m}^{-1}$

45-54 $\mathrm{T} \mathrm{m}^{-1}$

7 total

6 rebunching

7 zero degree

4-6 (rebunching)

7-17 (zero degree) four cavities are surrounded by external triplet lenses. The main linac parameters are summarized in Table V.

The KONUS beam dynamics design of this linac consists of a total of seven KONUS sections. As in the previous example, the first KONUS section omits the rebunching section and starts with a zero degree section, since the longitudinal matching is provided by a buncher cavity in the matching section in front of the linac. The transverse focusing lattice is optimized for a high beam current and low emittance growth. The phase advance per section (including space charge) is $80^{\circ}$ in the first sections and decreased to $60^{\circ}$ towards higher energies. The resulting transverse beam motion is shown in Fig. 25.

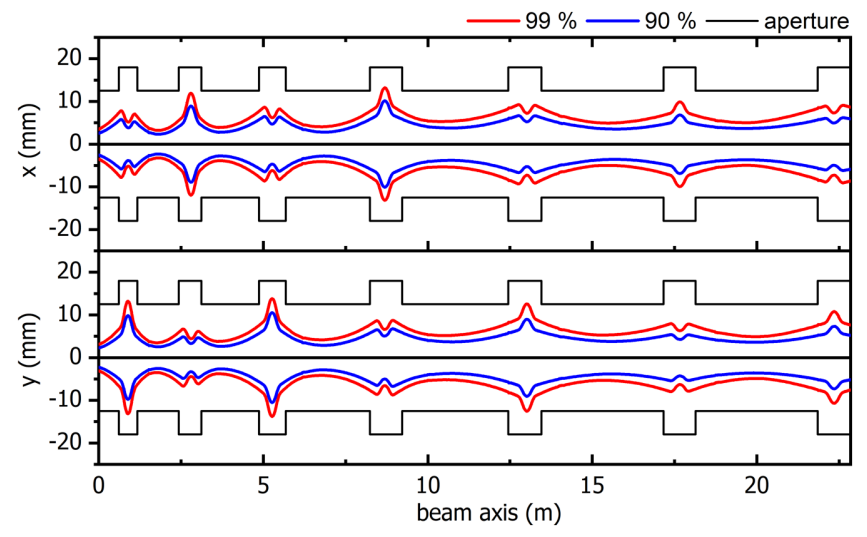

FIG. 25. Transverse beam envelopes along the linac.
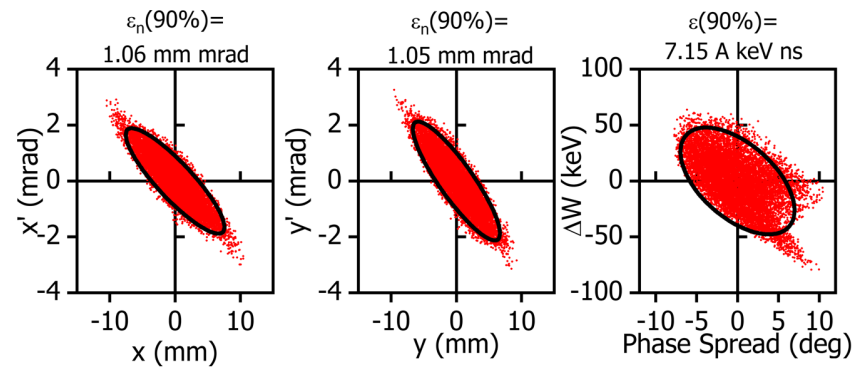

FIG. 26. Transverse and longitudinal particle distributions at the linac exit.
TABLE VI. Beam output parameters of the $85 \mathrm{MV}$ heavy ion linac.

\begin{tabular}{lc}
\hline \hline Beam current & $15 \mathrm{~mA}$ \\
Trans. emittance $\varepsilon_{n, \mathrm{rms}}$ & $0.23 \mathrm{~mm} \mathrm{mrad}$ \\
Long. emittance $\varepsilon_{\mathrm{rms}}$ & $1.75 \mathrm{~A} \mathrm{keV} \mathrm{ns}$ \\
Exit energy & $11.4 \mathrm{~A} \mathrm{MeV}$ \\
\hline \hline
\end{tabular}

Emittance growth along the whole linac is only $22 \%$ in the transverse planes and $13 \%$ in the longitudinal plane. The corresponding output particle distributions are displayed in Fig. 26, showing the preserved beam quality behind the linac, especially in the longitudinal plane. The output beam parameters are stated in Table VI.

The stability of a linac design is a major concern for real world operation. Therefore, the influence of a multitude of possible disturbing factors on the beam dynamics has to be investigated. To this end, statistical error studies of the beam dynamics are performed to evaluate the tolerances for errors, such as the misalignment of components during manufacturing and linac assembly, amplitude errors during operation, or fluctuations of beam properties. In this paper, we will focus on the most important part of these investigations, namely, the misalignment of the magnetic quadrupole lenses in the linac, since these errors prominently drive losses and emittance growth in an accelerator.

Error studies simulations were performed with TRACEWIN using $10^{7}$ macroparticles per error step with Gaussian error distributions. Stated errors are $2 \sigma$ of the Gaussian error distribution.

Figures 27-30 show the additional transverse rms emittance growth and the average losses for increasing displacement and rotation of the individual singlet lenses and the quadrupole triplets, respectively. These simulations have led to some remarkable results.

The additional emittance growth due to errors is almost negligible for all cases except for the rotation of singlets. In that case, the rotation of individual singlets around the beam axis ( $z$ axis) leads to significant emittance growth. Concerning particle losses, the rotation of completed triplets around the horizontal and vertical axes is very sensitive and can lead to significant losses, while the

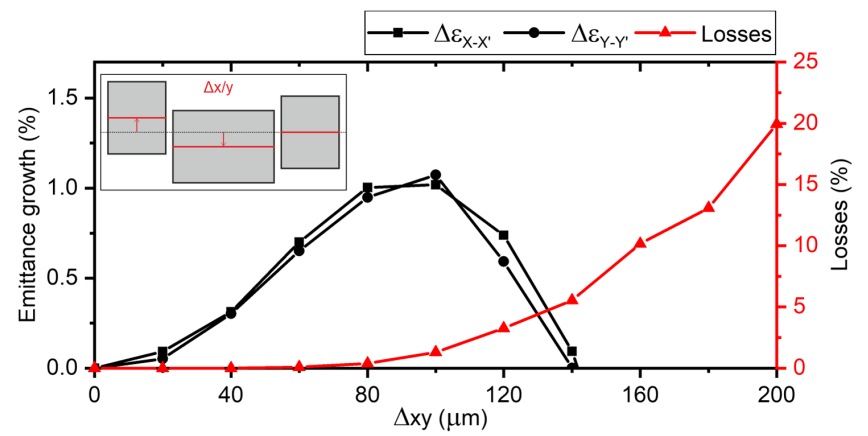

FIG. 27. The rms emittance growth and average losses for quadrupole singlet displacement along the linac. 


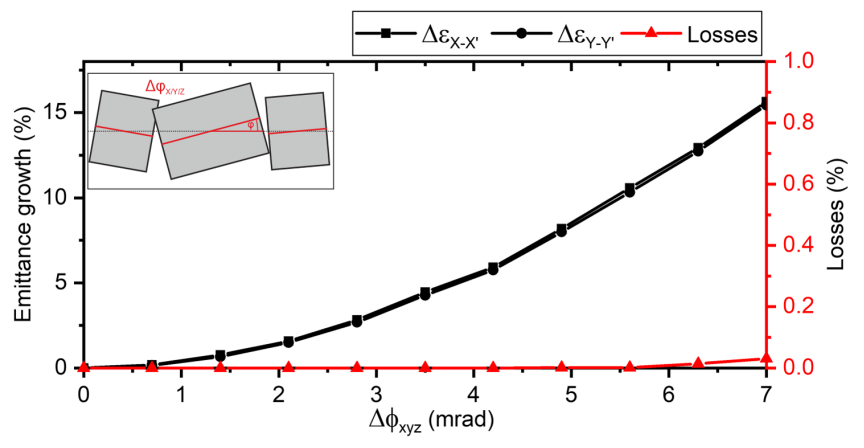

FIG. 28. The rms emittance growth and average losses for quadrupole singlet rotation around all three axes along the linac.

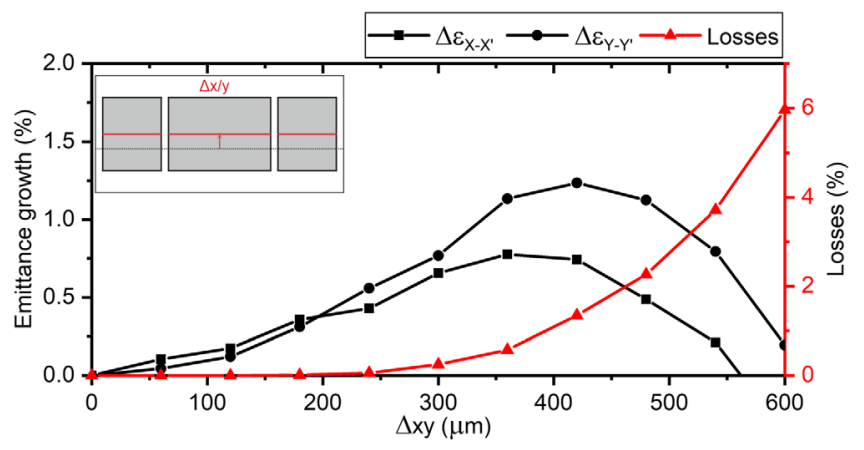

FIG. 29. The rms emittance growth and average losses for quadrupole triplet displacement along the linac.

rotation of individual singlets around transverse axes does not result in any significant losses.

Additionally, the displacement of quadrupole singlets and quadrupole triplets as a whole show very different sensitivities to errors. The simulations show that the displacement of quadrupole singlets in a triplet lens is much more sensitive and can lead to beam losses much earlier than triplet displacement as a whole. In both cases, emittance growth is finally limited to a small amount due to particle losses, which mostly reduce the halo of the beam.

Technically, the tolerance limit below $100 \mu \mathrm{m}$ to be reached for the precision of transverse singlet alignment (Fig. 27) is most challenging. An error below $2 \mathrm{mrad}$ in the transverse alignment of the completed triplet (Fig. 30) can

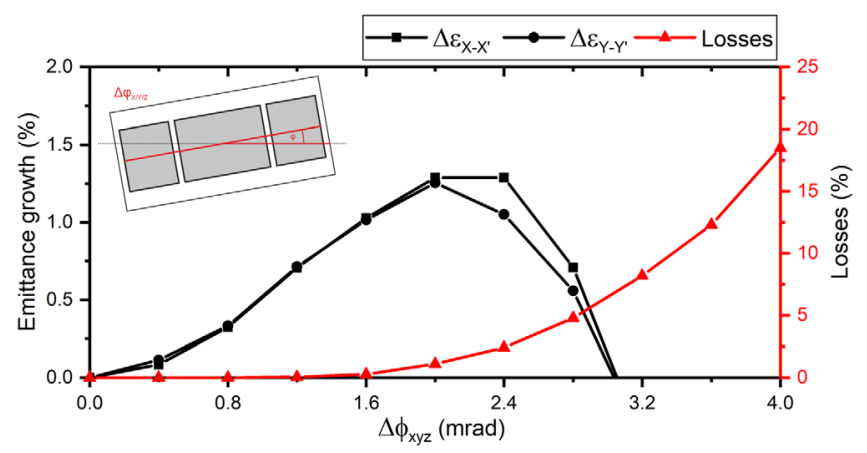

FIG. 30. The rms emittance growth and average losses for quadrupole triplet rotation around all three axes along the linac. be reached much easier, as the triplet length is more than $450 \mathrm{~mm}$ in this design.

\section{Comparison between KONUS, APF, and EQUUS}

Recently, IH structures applying alternating phase focusing (APF) $[31,32,33]$ were realized successfully to serve as synchrotron injectors for tumor therapy [34]. A comparison between KONUS and APF layouts was reported in Ref. [35]. APF's advantage is the missing need of magnetic lenses, which reduces structure costs by about $30 \%$. On the other hand, the emittance growths in all planes are larger at identical input emittances, and the beam current limits are reduced to about $50 \%$ against a KONUS design. The APF structure needs a slightly larger rf amplifier at a comparable structure length and voltage gain.

In the case of low-energy superconducting multicell cavities, the KONUS principle was modified into the equidistant multigap structure (EQUUS): Instead of a zero degree synchronous phase structure, these cavities show a constant cell length corresponding to the center particle velocity in the cavity midplane [36]. At the entrance and exit, particle bunches move at negative rf phases, while along the middle part of the cavity, rf phases are close to zero. This modification was invented for technical reasons. First beam results have been promising [37].

\section{Conclusions from simulation results}

The above presented examples show that KONUS beam dynamics can be particularly effective for challenging linac projects. While the roots of KONUS beam dynamics lie in heavy ion acceleration, as demonstrated in case B for high beam currents, it can also be applied very effectively to high-current proton acceleration (case A).

A main outcome of the error studies in both cases with respect to quadrupole tolerances can be summarized in the following way. (i) The most severe error is a transverse singlet displacement: They should be kept well below $\pm 0.1 \mathrm{~mm}$ in typical linac layouts. (ii) In the case of triplet focusing, the completed triplet has significantly higher tolerances of transverse misalignment against the beam axis.

This is a plus for triplet focusing like in KONUS: The tolerances within each triplet can be kept very low and are guaranteed by the manufacturer and controlled by the final acceptance tests. However, the short- and long-term stability of the installed accelerator elements can be hardly estimated, and control measurements are quite difficult in cases where the lenses are integrated into cavities. The error analysis in cases $\mathrm{A}$ and $\mathrm{B}$ results in tolerable transverse misalignments of the triplet lenses as big as \pm 0.3 to $\pm 0.5 \mathrm{~mm}$.

The development of the KONUS beam dynamics has been driven by heavy ion accelerator projects and by making optimum use of H-type cavities for that purpose. While the advantage of very high shunt impedance at low beam velocities is undoubted, the competitiveness with respect to beam quality is still under discussion from time to time when 
compared with convenient ion linac designs. It is admitted that FODO quadrupole focusing combined with a negative synchronous phase structure gives the best beam quality when parameter errors are put to zero. However, in many applications a low content of halo particles around the wellconserved beam core is a minor problem or can be separated by beam collimators in front of the beam application. Other arguments in favor of H-type linacs in combination with KONUS beam dynamics are the compactness of these designs in all three dimensions, the low rf power losses, and the robustness against parameter errors. The layouts discussed in Secs. VA and V B are significantly shorter than all existing rf linacs with comparable beam features.

\section{SUMMARY AND OUTLOOK}

This article explains for the first time the concept of the KONUS beam dynamics in detail and demonstrates the high-current beam capabilities including an error study. Besides a simplified analytical approach to describe the dynamics along a zero degree section, a matrix formalism was applied to analyze the transverse beam dynamics along a full KONUS period. The results are plotted in a stability chart corresponding to that of Smith and Gluckstern in the case of negative synchronous structures with a FODO lattice. Sections III and IV explain how to estimate the number of gaps per KONUS period when the beam input parameter set, drift tube aperture, and gap voltage gain are given. Handy transport matrices serving as good approximations for compact triplet lenses with negligible drifts between singlets have been presented. The simulations in Sec. V give detailed insight into design examples of linacs based on $\mathrm{H}$-mode cavities with KONUS beam dynamics and including space charge.

Future developments aim to further improve the beam simulation tools. In consequence, it should be possible to design KONUS structures with improved features like improved beam quality. Progress in production techniques will allow one to develop cavities with increased rf operation frequencies and with higher surface and acceleration fields.

Other topics for an improvement of the overall linac performance will be advanced transport section designs from the RFQ into the DTL and from the DTL to the following beam application-especially under high current conditions-as well as a better integration of steerers and beam diagnostics.

\section{ACKNOWLEDGMENTS}

We like to thank Professors Haruhiko Morinaga, Ecke Nolte, Paul Kienle and Dr. Norbert Angert from TU Munich and GSI Darmstadt, who gave always their support during the development of this novel type of ion accelerator. Important additional funding came from Grants No. BMBF06F134I, No. BMBF06FY161I, No. BMBF05P09RFRB8, No. BMBF05P12RFRB9.

\section{APPENDIX: BEAM DYNAMICS CODE LORASR}

KONUS needs an adequate simulation code to support an easy and transparent design of every single KONUS period. LORASR was developed and extended since 1982 [2]. The designs were focused on low-current, heavy ion acceleration at the beginning. Later on, high-current heavy ion beams for heavy ion driven inertial fusion (HIDIF) [1] were investigated. The first realized high-current machine designed with LORASR was the high-current injector HSI at GSI-Unilac [17]. In the case of $\mathrm{U}^{4+}$ from $120 \mathrm{~A} \mathrm{keV}$ to $1.4 \mathrm{~A} \mathrm{MeV}$, the simulated current limit is $20 \mathrm{~mA}$. The $70 \mathrm{~mA}$ FAIR proton linac from 3 to $68 \mathrm{MeV}$ is the first application of KONUS on a high-current proton-DTL project [24]. Two KONUS design cases developed with LORASR and fine-tuned by TRACEWIN are described in detail in Sec. V.

Table VII shows in the left column the input parameters needed to design an individual KONUS section. LORASR defines then the array and geometry of acceleration gaps. Every gap field is approximated by an axially symmetric field. Linearized longitudinal fields are defined for four radial zones, which are divided along the beam axis into five individual zones, resulting in a total of 20 squares. Each particle is tracked in a 30-step process along each gap. Space charge calls are possible after each gap transport. The fast Fourier transform (FFT) with circular boundary conditions is the standard space charge routine [26], while particle-particle interaction can be activated on demand for low particle numbers (below 104). Quadrupoles and solenoids are foreseen as focusing elements. Standard effective field approximations are used to take fringe field effects into account. The right column in Table VII shows the LORASR output parameters. The LORASR standard plots are (i) $X \%$ envelopes in the $x z-, y z-, W_{p s} z-$ and $\phi_{p s} z-$ planes; (ii) $X \%$ envelopes in the $W_{p c} z-$ and $\phi_{p c} z$ - planes; (iii) $100 \%$ envelopes with a particle loss histogram along the $z$ axis; (iv) center particle motion in the $W_{c s} \phi_{c s}$ - plane; (v) input and output particle cluster plots in the $x x^{\prime}, y y^{\prime}$, and $z z^{\prime}$ planes, including an ellipse, which contains $X \%$ of all particles and plotting the related normalized effective emittance value; (vi) relative, normalized emittance growths along the $z$ axis in all three planes; and (vii) phase advances for each complete KONUS section.

A convergence and benchmark status of LORASR with other codes is reported in Ref. [38].

Error studies can be performed by defining various types of machine errors for the different beam line elements [39].

The following error types are available in LORASR: (i) magnetic lens (quadrupole or solenoid) translations $\Delta X_{i}$ and $\Delta Y_{i}$ (each element $i$ separately); (ii) lens rotations $\Delta \varphi_{x}, \Delta \varphi_{y}$, and $\Delta \varphi_{z}$ (each element $i$ separately, e.g., singlets, or user-defined lens arrays, e.g., doublets or triplets); (iii) voltage amplitude errors $\Delta U_{j, i}$ for single gaps ( $i$ ) and whole cavities ( $j$ ); (iv) cavity phase errors $\Delta \varphi_{j}$ 
TABLE VII. Input and output parameters of the LORASR code.

\section{LORASR input parameters}

Drift to the entrance gap center

Number of gaps, effective gap voltages, and synchronous particle rf phases

Length ratios "drift tube/period" and "gap length / aperture diameter"

Energy and phase for the synchronous and the center particles at the first gap center

Input particle distribution around the center particle at the drift entrance

Location, polarity, eff. length, aperture, and field gradient for quadrupole singlets

Location, eff. length, aperture, and field for solenoids

Location, eff. length, and aperture for steerers

\section{LORASR output parameters}

List of periodic lengths, synchronous energies, gap and drift tube lengths, and inner diameters

Transit time factors for each radial gap zone and maximum on-axis electric field

Relative rf phase against a previous KONUS section to get the defined synchronous particle phases (readjustment of the drift length in the case of sections within the same cavity needed)

$W_{c s}$ and $\varphi_{c s}$ at the section exit

Number of lost particles by wall contact or relative energy deviation below a defined ratio applied at the entrance of cavity $j$; and (v) input beam errors, namely, transverse displacement and steering angle, energy, and phase offsets.

The error values are randomly distributed run by run (Gaussian distribution function truncated at the $2 \sigma$ width). For error studies, many runs (100-1000 typically) are needed for good statistics. Additionally, LORASR error settings can be defined explicitly for single runs, for instance, if measurement data from manufacturing or component alignment are available.

For the evaluation of the machine error study results, a couple of error analysis tools with the corresponding plots are available in LORASR. These are (i) loss profile plots for single runs, as well as for all runs (showing the averaged losses); (ii) plot of the maximum beam envelope including all runs, together with the envelope of the nominal case; (iii) probability distribution of the maximum aperture filling factor; and (iv) distribution of the additional relative emittance growth, compared to the nominal run.

The first three items of the above list are well suited for detecting bottlenecks of the beam dynamics design and, thus, to decide about corrective actions like steering corrections (number and positions of steering magnets). Moreover, by comparing the loss budgets resulting from different error settings, manufacturing and alignment tolerances can be established.

The last item of the above list allows one to localize the beam degradation caused by machine errors.

[1] U. Ratzinger and R. Tiede, Status of the HIIF RF linac study based on H-mode cavities, Nucl. Instrum. Methods Phys. Res., Sect. A 415, 229 (1998).
[2] U. Ratzinger, Effiziente Hochfrequenz-Linearbeschleuniger für leichte und schwere Ionen, Habilitationsschrift (Goethe-Universität, Frankfurt, 1998).

[3] T. P. Wangler, $R F$ Linear Accelerators (Wiley-VCH, Berlin, 2008).

[4] U. Ratzinger, R. Geier, W. Schollmeier, S. Gustavsson, and E. Nolte, The three-harmonics double-drift buncher at the Munich heavy ion postaccelerator, Nucl. Instrum. Methods Phys. Res. 205, 381 (1983).

[5] U. Ratzinger, E. Nolte, R. Geier, N. Gärtner, and H. Morinaga, The upgraded Munich linear heavy ion postaccelerator, Nucl. Instrum. Methods Phys. Res., Sect. A 263, 261 (1988).

[6] U. Ratzinger, A low beta rf linac-structure of the IH-type with improved radial acceptance, in Proceedings of the 1988 Linear Accelerator Conference (LINAC'88) Newport News, VA (JACoW, Geneva, 2003), p. 185.

[7] N. Angert, L. Dahl, J. Glatz, J. Klabunde, M. Mueller, and B. Wolf, UNILAC modifications for an improved synchrotron injector performance, in Proceedings of the 2nd European Particle Accelerator Conference (EPAC'90) Nice, France (JACoW, Geneva, 1990), p. 503.

[8] J. Klabunde, The high charge state injector for GSI, in Proceedings of the 1992 Linear Accelerator Conference (LINAC'92) Ottawa, Canada (JACoW, Geneva, 2013), p. 570.

[9] U. Ratzinger, The IH-structure and its capability to accelerate high current beams, in Proceedings of the 1991 Particle Accelerator Conference, San Francisco, CA, 1991 (IEEE, New York, 1991), p. 572.

[10] H. D. Haseroth, $\mathrm{Pb}$ injector at CERN, in Proceedings of the 18th International Linear Accelerator Conference (LINAC'96) Geneva, Switzerland (CERN, Geneva, 1996), p. 283.

[11] O. Kester et al., Accelerated radioactive beams from REXISOLDE, Nucl. Instrum. Methods Phys. Res., Sect. B 204, 20 (2003). 
[12] J. G. Alessi et al., Construction of the BNL EBIS preinjector, in Proceedings of the 23rd Particle Accelerator Conference, Vancouver, Canada, 2009 (JACoW, Geneva, 2009), p. 407.

[13] B. Schlitt, Commissioning and operation of the injector linacs for HIT and CNAO, in Proceedings of the 24th Linear Accelerator Conference (LINAC'08) Victoria, Canada (JACoW, Geneva, 2008), p. 720.

[14] A. V. Butenko et al., The heavy ion injector at the NICA project, in Proceedings of the 27th Linear Accelerator Conference (LINAC'14) Geneva, Switzerland (JACoW, Geneva, 2014), p. 1068.

[15] L. A. Dahl et al., The HITRAP decelerator project at GSI-Status and commissioning report, in Proceedings of the 24th Linear Accelerator Conference (LINAC'08) Victoria, Canada (JACoW, Geneva, 2008), p. 100.

[16] R. E. Laxdal, Design of a drift tube linac for the ISAC project at TRIUMF, in Proceedings of the 18th International Linear Accelerator Conference (LINAC'96) Geneva, Switzerland (CERN, Geneva, 1996), p. 435.

[17] U. Ratzinger, Commissioning of the new GSI high current linac and HIF related rf linac aspects, Nucl. Instrum. Methods Phys. Res., Sect. A 464, 636 (2001).

[18] L. Groening et al., Upgrade of the unilac for FAIR, in Proceedings of the 6th International Particle Accelerator Conference (IPAC'15) Richmond, VA (JACoW, Geneva, 2015), p. 1281.

[19] G. Clemente, U. Ratzinger, H. Podlech, L. Groening, R. Brodhage, and W. Barth, Development of room temperature crossbar-H-mode cavities for proton and ion acceleration in the low to medium beta range, Phys. Rev. Accel. Beams 14, 110101 (2011).

[20] R. Billinge et al., Concept for a lead-ion accelerator facility at CERN, CERN Yellow Report No. CERN 90-01.

[21] A. Angert et al., CERN heavy-ion facility design report, CERN Yellow Report No. CERN 93-01.

[22] J. Broere, H. Kugler, M. Vretenar, U. Ratzinger, and B. Krietenstein, High power conditioning of the $202 \mathrm{MHz} \mathrm{IH}$ tank 2 at the CERN linac3, in Proceedings of the 19th International Linac Conference (LINAC'98) Chicago, IL (ANL, Argonne, Illinois, USA, 1998), p. 771.

[23] C. Zhang, H. Podlech, U. Ratzinger, and R. Tiede, Efficient solution for accelerating very high intensity beams in the low and medium energy regime, Phys. Rev. Accel. Beams 22, 100101 (2019).

[24] R. Tiede et al., Improved beam dynamics and cavity rf design for the FAIR proton injector, in Proceedings of the 28th Linear Accelerator Conference (LINAC'16) East Lansing, MI (JACoW, Geneva, 2016), p. 111.

[25] L. Smith and R. L. Gluckstern, Focusing in linear ion accelerators, Rev. Sci. Instrum. 26, 220 (1955).
[26] R. Tiede et al., LORASR code development, in Proceedings of the 10th European Particle Accelerator Conference, Edinburgh, Scotland, 2006 (EPS-AG, Edinburgh, Scotland, 2006), p. 2194.

[27] D. Uriot and N. Pichoff, Status of TRACEWIN code, in Proceedings of the 6th International Particle Accelerator Conference (IPAC'15) Richmond, VA (JACoW, Geneva, 2015), p. 92.

[28] C. Kleffner et al., Status of the FAIR proton linac, in Proceedings of the 29th Linear Accelerator Conference (LINAC'18) Beijing, China (JACoW, Geneva, 2018), p. 787.

[29] M. Schuett, Development of a $325 \mathrm{MHz}$ Ladder RFQ, Ph.D. thesis, Goethe University, Frankfurt, Germany, 2018.

[30] H. Hähnel, Development of an IH-type linac for the acceleration of high current heavy ion beams, Ph.D. thesis, Goethe University, Frankfurt, Germany, 2017.

[31] D. Boussard, Radiofrequency Focusing in Heavy Ion Linear Accelerators, in Linear Accelerators, edited by P. Lapostolle and Septier (North Holland, Amsterdam, 1970).

[32] H. Deitinghoff, P. Junior, and H. Klein, Properties of heavy ion linacs with alternating phase focusing, in Proceedings of the 1976 Proton Linear Accelerator Conference (LINAC'76) Chalk River, Canada (JACoW, Geneva, 2013), p. 238.

[33] D. A. Swenson, Alternating phase focused linacs, Part. Accel. 7, 6 (1976).

[34] Y. Iwata et al., Alternating phase focused IH-DTL for heavyion medical accelerators, in Proceedings of the 10th European Particle Accelerator Conference, Edinburgh, Scotland, 2006 (EPS-AG, Edinburgh, Scotland, 2006), p. 2328.

[35] S. Minaev, U. Ratzinger, and B. Schlitt, APF or KONUS drift tube structures for medical synchrotron injectors-A comparison, in Proceedings of the 18th Particle Accelerator Conference, New York, 1999 (IEEE, New York, 1999), p. 3555.

[36] S. Minaev, U. Ratzinger, H. Podlech, M. Busch, and W. Barth, Superconducting, energy variable heavy ion linac with constant $\beta$, multicell cavities of $\mathrm{CH}$ type, Phys. Rev. Accel. Beams 12, 120101 (2009).

[37] W. Barth et al., First heavy ion beam tests with a superconducting multigap cavity, Phys. Rev. Accel. Beams 21, 020102 (2018).

[38] I. Hofmann and W. Chou, editors, ICFA Beam Dynamics Newsletter No. 41, 2006, http://icfa-bd.kek.jp/Newsletter41 .pdf.

[39] R. Tiede et al., Improvements of the LORASR code and their impact on current beam dynamics designs, in Proceedings of the 27th Linear Accelerator Conference (LINAC'14) Geneva, Switzerland (JACoW, Geneva, 2014), p. 569. 